\title{
Chants en cascade. Une acoustémologie de la résonance du lieu au Bosavi, Papouasie-Nouvelle-Guinée
}

Waterfalls of Song. An Acoustemology of Place Resounding in Bosavi, Papua New Guinea

\section{Steven Feld}

Traducteur : l'anglais (États-Unis) par Lila Lakehal

\section{OpenEdition}

\section{Journals}

Édition électronique

URL : https://journals.openedition.org/clo/8303

DOI : $10.4000 /$ clo.8303

ISSN : 2266-1816

Éditeur

INALCO

\section{Édition imprimée}

Date de publication : 30 juin 2020

Pagination : 23-85

ISBN : 978-2-85831-392-1

ISSN : 0396-891X

Référence électronique

Steven Feld, «Chants en cascade. Une acoustémologie de la résonance du lieu au Bosavi,

Papouasie-Nouvelle-Guinée », Cahiers de littérature orale [En ligne], 87 | 2020, mis en ligne le 16 septembre 2021, consulté le 09 novembre 2022. URL : http://journals.openedition.org/clo/8303 ; DOI : https://doi.org/10.4000/clo.8303

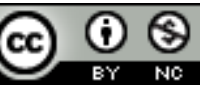

Creative Commons - Attribution - Pas d'Utilisation Commerciale 4.0 International - CC BY-NC 4.0 https://creativecommons.org/licenses/by-nc/4.0/ 


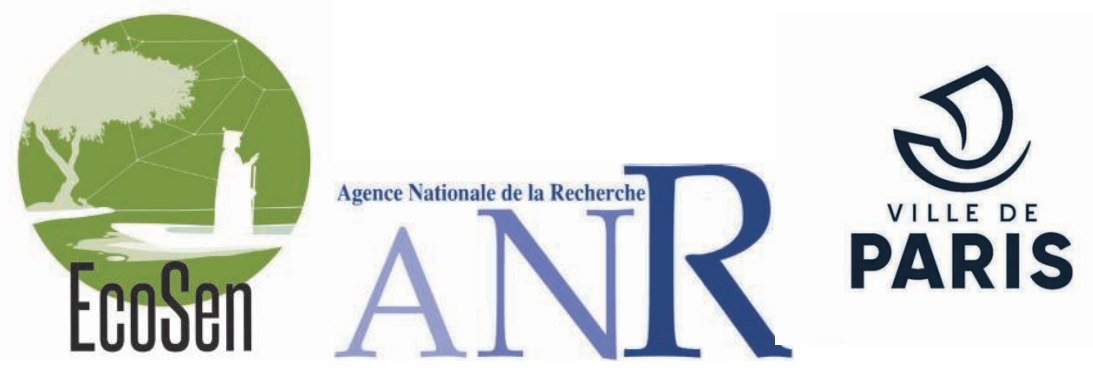




\title{
Chants en cascade. Une acoustémologie de la résonance du lieu au Bosavi, Papouasie-Nouvelle-Guinée ${ }^{1}$
}

\author{
Steven FELD \\ University of New Mexico \\ Traduction de l'anglais (États-Unis) par Lila LAKEHAL ${ }^{2}$
}

Le sens du lieu : l'expression est si commune que le terme «sens » passe presque complètement inaperçu ${ }^{3}$. Mais comment un lieu est-il vraiment senti ? Comment les engagements perceptifs que nous appelons sensoriels, sont-ils essentiels

1. Traduction de l'article de FeLD Steven, 1996, "Waterfalls of Song. An Acoustemology of Place Resounding in Bosavi, Papua New Guinea” in Feld Steven \& BAsso Keith H. (eds.), Senses of Place, School of American Research Press, Santa Fe, pp. 91-135.

2. La traductrice tient à remercier Mélanie Bourlet, Marie Lorin et Katell Morand de lui avoir confié la traduction inédite de cet article essentiel, et d'avoir offert la sagacité de leurs précieux commentaires tout au long du travail. Elle sait gré à Steven Feld de sa confiance et de sa disponibilité dans leurs échanges en ligne et lors des Rencontres interdisciplinaires « Ec(h)opoétiques » organisées par l'Inalco en mai 2019, au cours desquelles a résonné la subtile et puissante musicalité de ses recherches et de la poésie kaluli.

3. Si l'expression sense of place est en effet commune dans les littératures anglo-saxonnes en sciences sociales ainsi que dans le champ des politiques publiques, elle ne connait pas d'équivalent français immédiat. Nous avons donc privilégié sa traduction littérale en français, de manière à conserver le jeu polysémique récurrent dans l'ensemble de l'article, avec le ou les cinq sens. De manière plus générale, la langue de $S$. Feld fait la part belle aux jeux de mots fertiles, permis par la fluidité de la langue anglaise : nous avons tenté, autant que faire se peut, de rendre sensible (audible) dans la traduction, le style « sensuel » de l'auteur, qui revêt des implications tant poétiques qu'épistémologiques. L'ensemble des notes de bas de page de cet article est de la traductrice. 
dans les constructions conceptuelles du lieu ? Et comment cette sensualité riche de sentiments participe-t-elle à naturaliser le sens qu'on a d'un lieu ? Ces questions guident mon enquête sur la sensorialité et la sensualité à l'œuvre dans la dénomination et l'évocation poétique des lieux par le peuple kaluli du Bosavi, en Papouasie-Nouvelle-Guinée. Je souhaite mettre au jour un double mouvement réciproque : de même qu'un lieu est ressenti, les sens sont localisés; de même que les lieux font sens, les sens font lieu ${ }^{4}$. Dans la mesure où le son et un sensorium ${ }^{5}$ fondé sur l'ouie et la voix sont au cœur de l'expérience et de l'expression des Kaluli dans la forêt tropicale, l'objectif de cette exploration est d'interpréter une acoustémologie ${ }^{6}$, terme par lequel je désigne les conditions locales de la sensation acoustique, du savoir, et de l'imagination incorporés ${ }^{7}$ dans la résonance du lieu propre à la culture bosavi.

Ce chapitre commence par de brèves considérations sur la sensation, le son, la synesthésie et les soundscapes ${ }^{8}$ qui donnent le contexte du cadre général de mon enquête, une phénoménologie et une herméneutique sociales des sens du lieu. Après avoir montré en quoi la recherche sur l'expérience acoustique et l'expression du lieu est demeurée relativement peu développée, je présente le monde sonore du peuple kaluli. Ensuite, je propose deux parties ethnographiques portant sur l'acoustémologie de l'écoulement ${ }^{9}$. La première traite des pratiques de dénomination kaluli, afin de montrer comment le caractère inséparable des eaux et des terres de la forêt tropicale est expérimenté et imaginé comme le flot

4. Jeu de mots polysémique, cf. note précédente.

5. Ce terme technique, désignant l'environnement sensoriel toujours changeant perçu par un sujet, a été redéfini et employé au $\mathrm{Xx}^{\mathrm{e}}$ siècle par la théorie des médias développée par M. McLuhan, E. Carpenter et W. J. Ong (cf. infra).

6. Le terme acoustemology, qui associe l'acoustique et l'épistémologie, a été forgé par S. Feld pour signifier que le son est un chemin d'accès au savoir, aussi avons-nous directement transposé ce néologisme en français.

7. Embodied peut être traduit par « incarné », cependant compte tenu de l'importance du corps dans cette étude, nous avons privilégié la traduction « incorporé ».

8. Nous avons conservé le terme anglais de soundscape, dans la tradition issue des travaux de Murray Schafer. La notion de « paysage sonore » a une histoire différente en France (voir par exemple Corbin Alain, 1994, Les Cloches de la terre, paysage sonore et culture sensible dans les campagnes au XIX $X^{e}$ siècle, Albin Michel, Paris, 359 p.).

9. Le terme flow en anglais renvoie à la fois au terme flot, au processus et au flux généré. Nous avons privilégié la traduction « écoulement », qui suggère bien le rapport à l'eau, si important dans la suite de l'article, ainsi que la dimension progressive dans le temps de l'action, tout en lui substituant parfois, par souci de style, le terme « flot ». 
de la voix à travers les contours du corps. Ce trope de l'écoulement est alors étudié tel qu'il apparaît dans des textes poétiques de chants, dans lesquels le fait de chanter une séquence de noms de lieux emmène les auditeurs dans un voyage qui s'écoule le long des cours d'eau et à travers les terres locales. L'écoulement de ces sentiers poétiques chantés est lié émotionnellement et physiquement au flot sensuel de la voix chantante. Mettre en lien ces sentiers qui s'écoulent révèle une acoustémologie kaluli des relations aux lieux, une fusion de l'espace et du temps qui assemble les vies et les événements en tant que souvenirs incorporés. Les pouvoirs évocateurs de cette acoustémologie atteignent un sommet esthétique dans la performance poétique, là où l'écoulement expressif de la voix fusionne avec l'écoulement vécu des noms de lieux chantés, afin de créer des chants en cascade, un sens de la résonance du lieu.

\section{Sens, incorporation, synesthésie}

« La perception [...] ne m’offre pas des vérités comme la géométrie, mais des présences » (Merleau-Ponty, 1964, p. 14). Quelles sont ces « présences » qui sont données dans la perception ? Merleau-Ponty insistait sur le fait qu'elles étaient avant tout les présences des corps qui ressentent et perçoivent, des corps dont l'expérience sensorielle n'était jamais totalement sublimée en connaissance abstraite. Les sensations, assurait-il, étaient toujours des présences vécues, les présences de ce que les psychologues et philosophes cognitivistes appellent un « esprit incorporé » (Varela et al., 1991), ou un « corps dans l'esprit » (Johnson, 1987).

Cependant, les sens, les « surfaces sensori-motrices » du corps, ne se limitent pas à des présences incorporées, et ils représentent davantage que des espaces d'expérience pour établir des points et des lieux de contact physique et social (Straus, 1963). Dans The Absent Body (1990), Drew Leder développe cet axe de critique en s'interrogeant sur la raison pour laquelle, alors que le corps est si essentiel à l'expérience sensorielle, et qu'il situe si activement le sujet, il serait aussi absent de l'expérience ou hors du focus. Pourquoi le corps n'est-il pas l'objet thématique direct de l'attention et de l'expérience, et pourquoi est-il en recul par rapport à l'expérience directe ? Leder développe ces questions en répondant à l'observation de Merleau-Ponty, faite dans La Structure du comportement (1963), selon laquelle « être situé depuis un certain point de vue implique nécessairement de ne pas voir ce point de vue » (Leder, 1990, p. 12). Il poursuit : «Cela constitue le complément nécessaire de la description gestaltiste figure/ fond de la perception. Comme Merleau-Ponty l'écrit [dans Phénoménologie de la perception, 1962], "le corps propre est le troisième terme, toujours sous-entendu, 
de la structure figure et fond, et toute figure se profile sur le double horizon de l'espace extérieur et de l'espace corporel" » (Leder, 1990, p. 13).

L'hypothèse de Leder pour expliquer pourquoi certaines dimensions du corps sont toujours expérimentées à l'avant-plan, tandis que d'autres sont repoussées à l'arrière-plan, repose sur la même « gestalt de type figure et fond pour caractériser non seulement le champ d'expérience du corps, mais aussi la structure même du corps vivant l'expérience » (Leder, 1990, p. 24). Il assure que « ces modes d'absence proviennent directement de la structure fondamentale de l'incorporation », caractérisant plus loin « le corps vécu comme un être extatique/récessif, engagé à la fois dans un bond vers l'extérieur et un recul vers l'arrière. À travers sa surface sensori-motrice, il se projette dans le monde. En même temps, il s'éloigne de sa propre appréhension dans des profondeurs viscérales anonymes. Le corps n'est jamais une simple présence, mais ce qui est éloigné de lui-même, un être de différence et d'absence » (Leder, 1990, p. 103 ; voir aussi Levin, 1985 ; Schilder, 1950).

Établir ces présences et absences complexes et multiples du corps implique une autre structure interactive de type figure et fond, celle des sens. L'expérience vécue entraîne des changements constants dans les figures et fonds sensoriels, des capacités constantes d'interactions ou de correspondances multi- ou inter-sensorielles. Les jeux de figure et fond, où un sens surgit alors même qu'un autre recule, et où les positions de domination et de subordination s'échangent ou se mélangent, se fondent en une synesthésie, « la transposition d'images sensorielles ou d'attributs sensoriels d'une modalité à une autre » (Marks, 1978 , p. 8). La synesthésie souligne la complexité des ratios sensoriels, les riches connections inhérentes à la multiplicité des sources de sensations, les résonances fourmillantes et les réverbérations corporelles qui émergent de perceptions simultanées (Cytowic, 1989). Ce « palimpseste des sens déteignant dans les horizons d'attente les uns des autres »(Taussig, 1993, p. 57) révèle comment « le champ synesthésique, comme le champ métaphorique en général, élargit l'horizon du savoir en rendant réelles des significations qui étaient auparavant seulement potentielles » (Marks, 1978, p. 254). Dans son ouvrage Mimesis and Alterity, Taussig estime que ce potentiel métaphorique et synesthésique rappelle la mimesis, « le pouvoir magique de l'imitation [...] dans laquelle la représentation transmet ou tire son pouvoir de ce qui est représenté » $(1993$, p. 2). Ce même potentiel métaphorique et synesthésique rappelle également l'iconicité ${ }^{10}$, ou

10. Traduction littérale du terme iconicity, employé en linguistique et sémiotique pour désigner la relation de similarité entre la forme et la signification d'un signe. 
les manières par lesquelles ce qui perçoit et ce qui est perçu se fondent et se mêlent à travers le contact sensuel, expérimentant des ressemblances internes qui se font écho, vibrent, et persistent comme des traces d'un mode sensoriel à un autre, présentes à un certain niveau et absentes à d'autres, reliant continûment l'expérience corporelle à la pensée et à l'action (Feld, 1988; Jackson, 1989, p. 119-155; Ohnuki-Tierney, 1991).

Mais la sensation, la présence sensuelle, est toujours plus que l'incorporation, plus que les structures perceptives de type figure et fond, plus que le potentiel pour la synesthésie. C'était l'intuition de Henri Bergson, il y a longtemps dans Matière et mémoire, pour lequel « il n'y a pas de perception qui ne soit chargée de souvenirs. Aux données immédiates et présentes de nos sens, nous mêlons des milliers de détails issus de notre expérience passée » (1988, p. 33). Par conséquent, « ce que vous avez donc à expliquer, [...] n'est pas comment la perception naît, mais comment elle se limite, puisqu'elle serait, en droit, l'image du tout, et qu'elle se réduit de fait à ce qui vous intéresse » (1988, p. 40). Le problème soulevé par Bergson - relier le corps en activité comme lieu de passage du processus de fabrication de la mémoire - est développé dans le livre Remembering d'Edward Casey (1987). Ce dernier écrit :

En se déplaçant vers ou à travers un lieu donné, le corps importe son propre passé localisé dans son expérience présente : son histoire locale est littéralement une histoire de lieux. Cette importation même des lieux passés survient à mesure que le corps établit la direction, la hauteur et la distance, et influence en effet ces dernières d'une myriade de manières. L'orientation dans un lieu (qui est établie par ces trois facteurs) ne peut pas être continûment affectée de novo, mais émerge de l'ombre toujours grandissante de notre passé corporel. (Casey, 1987, p. 194)

Parce que le mouvement peut s'appuyer sur le jeu kinesthésique des sens du toucher, de l'ouie et de la vision, la localisation implique toujours l'intrication de la présence corporelle sensuelle et de l'engagement perceptif. 


\section{Paysage, espace acoustique, soundscape $e^{11}$}

Étant donné la dimension éminemment multi-sensorielle de l'expérience perceptive, on s'attendrait à trouver une conceptualisation multi-sensorielle du lieu. Mais de manière générale, les travaux d'ethnographie et de géographie culturelle sur les sens du lieu ont été dominés par l'approche visuelle, profondément ancrée dans le concept européen de paysage. Denis Cosgrove a analysé comment deux notions distinctes de paysage, partageant toutes deux une approche visuelle dominante, ont fusionné en Occident. Dans le premier cas, depuis plus de quatre cents ans,

l'idée du paysage en est venue à décrire la représentation artistique et littéraire du monde visible, la scène (littéralement ce qui est vu) telle qu'elle est vue par un spectateur. Cela impliquait une sensibilité particulière [...] étroitement liée à une dépendance grandissante envers la faculté visuelle comme le moyen par lequel on pouvait atteindre la vérité : « il faut le voir pour le croire ». Des innovations techniques importantes pour représenter cette vérité incluaient la perspective à point de fuite unique et l'invention d'auxiliaires à la vue comme le microscope, le télescope, et la caméra. (Cosgrove, 1984, p. 9)

Dans le deuxième cas, celui du paysage comme notion incorporée dans les problématiques analytiques de la géographie académique, le concept « dénote l'intégration de phénomènes naturels et humains qui peuvent être vérifiés empiriquement et analysés par les méthodes de l'enquête scientifique sur une portion délimitée de la surface terrestre »(Ibid.). Cosgrove estime que ces deux sens du paysage «sont intimement liés à la fois historiquement et comme manière commune de s'approprier le monde par l'objectivité accordée à la faculté de la vue et sa technique dérivée de représentation picturale » (Ibid.).

Mais qu'en est-il du lieu tel qu'il est entendu et ressenti ? Du lieu tel que sonnant ou résonnant ? Contrastant avec la longue histoire de l'idée de paysage dans les recherches et les représentations artistiques comme scientifiques, les études sur les façons dont les mondes sont appréhendés de manière sonore ont une

11. Soundscape se distingue ici de landscape, paysage au sens généralement compris comme visuel du terme, que nous ne qualifierons pas ici à des fins de simplicité. Pour la distinction entre soundscape et paysage sonore, voir note $\mathrm{n}^{\circ} 8$. 
histoire plus limitée. En soutenant l'idée selon laquelle le « bouche-à-oreille ${ }^{12} \gg$ de l'expérience auditive et orale n'a jamais bénéficié de la même primauté, en tant que preuve et représentation, que l'《intuition ${ }^{13} \gg$ visuelle, Edmund Carpenter et Marshall McLuhan ont introduit la notion d' « espace acoustique » dans leur revue Explorations (1953-1959). Le terme provient de leurs projets au Center for Culture and Technology de l'université de Toronto sur les transformations des médias, tout particulièrement les manières de réinterpréter l'histoire de l'oralité et de l'écriture du point de vue des communications électroniques au $\mathrm{Xx}^{\mathrm{e}}$ siècle. Dans ce contexte, l'article de Carpenter sur l'espace acoustique fut le premier à décrire les implications culturelles d'un « point d'audition ${ }^{14}$ » à l'orientation simultanée et diffuse, sa proposition d'alternative au «point de vue » (1960). Ses études suivantes $(1971,1973,1980)$ en vinrent à associer espace acoustique et jeux entre vision et audition : ainsi l'expérience Inuit de l'espace dynamique et sphérique en Arctique était-elle liée aux imaginaires et procédés artistiques locaux, en particulier les jeux visuels et les descriptions du mouvement, de la profondeur et de la non-contention.

La notion d' « espace auditif » a également émergé au milieu des années 1950, dans un contexte entièrement différent. Le philosophe de la musique Victor Zuckerkandl (1956), s'inspirant de manière substantielle de la philosophie d'Henri Bergson et de Martin Heidegger, et de la psychophysique et psychologie perceptive et Gestalt de William James, Géza Révész et Erwin Straus, argumenta énergiquement contre l'idée selon laquelle la musique était uniquement une expérience du ton comme temps. Pour ce faire, il détailla les manières par lesquelles l'espace fusionne avec le temps de façon auditive, dans la progression et le mouvement des tons (1956, p. 267-348). Bien que cette interpénétration de l'espace et du temps de l'audition n'ait pas eu d'impact général sur les théorisations de l'espace et du lieu, Music and the External World, le premier volume de l'œuvre Sound and Symbol de Zuckerkandl (1956), a, de manière certaine, eu un impact décisif ailleurs, comme dans la critique philosophique

12. Nous avons traduit ainsi le terme hearsay, qui signifie également ouï-dire, rumeur, pour conserver le jeu de mots initial.

13. En anglais, le terme insight rend immédiatement sensible le lien au sens de la vue; en français, l'étymologie latine du mot «intuition », intueri, regarder attentivement, créé cette association.

14. Nous avons choisi de traduire l'expression earpoint par «point d'audition », pour conserver le parallélisme avec viewpoint « point de vue » et insister sur la capacité sensorielle. 
vigoureuse de Kathleen Higgins du platonisme en musique (Higgins, 1991), les explorations anthropologiques du symbolisme rituel, musical et sonore dans le travail d'Ellen Basso (1985) et de Paul Stoller (1989, p. 101-122), ainsi que dans les essais théoriques de Roy Wagner sur le symbole et la métaphore (1986).

De même que Zuckerkandl le musicien a influencé des anthropologues, Carpenter l'anthropologue a principalement influencé des musiciens. Quand le compositeur Murray Schafer organisa le World Soundscape Project à l'université Simon Fraser en 1970, les idées de Carpenter et de McLuhan, marginales à la fois dans l'anthropologie des arts et dans la géographie culturelle dans les années 1950 et 1960, furent introduites auprès de compositeurs et acousticiens dans un nouveau cadre, celui de l'étude de l'environnement sonore et de la communication acoustique. Le groupe de Schafer commença à enregistrer, observer et analyser de manière acoustique l'expérience sonore de l'espace et du lieu, particulièrement au Canada et en Europe, et développa un vocabulaire analytique, un système de notation, et un cadre comparatif pour l'étude de l'espace acoustique et de ses interprétations et réponses par l'être humain. Ce travail fut classé dans les rubriques générales de deux termes créés par Schafer, l'《écologie acoustique » et le «soundscape design ».

Schafer et ses collègues disséminèrent leurs idées à travers des médias allant des compositions musicales aux collages radiophoniques, et des rapports techniques aux journaux de voyage sous format papier ou cassette, ce qui aboutit à une synthèse générale, The Tuning of the World de Schafer (1977). Ce livre a suscité une grande attention envers les complexités acoustiques des environnements, particulièrement des pays du Nord, mais son impact a été surtout ressenti parmi les musiciens, les acousticiens, les architectes, et les artistes, compositeurs et preneurs de son de la radio (voir par exemple Schafer, 1993 ; Truax, 1984 ; Werner, 1992). L'écologie acoustique et les études de soundscape ont eu moins d'influence sur les ethnographes, susceptibles d'étudier comment les peuples entendent, répondent, et imaginent les lieux comme sensuellement sonores. D'un autre côté, la géographie humaniste, profondément influencée par les perspectives issues de la phénoménologie dans les années 1970 et 1980, a commencé à considérer les dimensions acoustiques du lieu avec moins de réserve (par exemple, Buttimer \& Seamon, 1977), mais les a rarement explorées, et jamais de la manière pleinement ancrée à même d'attirer l'attention de l'anthropologie.

Les travaux dans la lignée Carpenter-McLuhan-Schafer n’ont pas été pris au sérieux par les anthropologues; ils ont même été critiqués par ceux qui étaient les plus intéressés par ses conséquences pour l'analyse de problématiques liées aux sens et à la relation entre oralité et écriture (Feld, 1986 ; Finnegan, 1988, p. 139-174). En dépit de son intérêt affiché pour les ratios sensoriels, cette 
ligne de pensée a souvent réifié un grand partage entre le visuel et l'auditif, reproduisant une variante de l'idée selon laquelle « voir est une capacité analytique et réflexive. Le son est une capacité active et générative » (Schafer, 1985, p. 96). Une telle rhétorique simpliste a mené la plupart des ethnographes à détourner leur attention et suscité l'approche critique de Don Idhe, dont l'essai phénoménologique Listening and Voice pointait la vanité consistant à prendre le contrepied du centralisme visuel historique dans les discours analytiques occidentaux, en érigeant simplement un antivisualisme (1976, p. 21).

Ce que Idhe appelait de ses vœux à la place - un appel relayé récemment par l'anthropologue David Howes dans The Varieties of Sensory Experience (1991, p. 3-21, p. 167-191), était une réévaluation de tous les sens à partir de la perspective de leurs relations. C'est seulement ainsi, soutenaient Idhe et Howes, qu'une analyse sérieuse du son pourrait émerger d'une manière conforme à l'expérience vécue et à l'ethnographie. Étant donné les tentatives récurrentes d'essentialiser la vue comme une caractéristique de l'Occident (voir par exemple Ong, 1982), en opposition totale avec une centralité présumée du son, de l'odeur et du goût essentialisée pour des cultures « autres » non occidentales, une réévaluation des ratios sensoriels doit étudier de près comment la tendance au primat d'un sens sur les autres évolue toujours d'après le contexte selon l'emplacement du corps. Cette perspective nourrit ma position sur le son dans l'expérience sensorielle, particulièrement ses implications pour interpréter les mondes vivants du peuple kaluli en Papouasie-Nouvelle-Guinée.

\section{Vers une acoustémologie}

Si, en percevant, « notre corps entier vibre à l'unisson du stimulus [... alors] entendre est, comme toutes les perceptions sensorielles, une manière de saisir la réalité avec tout notre corps, nos os et viscères inclus » (Gonzalez-Crussi, 1989, p. 45 ; comparer avec Idhe, 1976, p. 81 et Ackerman, 1990, p. 186-190 pour les manières dont le son pénètre le corps). Le son, l'ouïe, et la voix marquent un nexus corporel de sensation et d'émotion spécifique, en raison de leur coordination avec le cerveau, le système nerveux, la tête, l'oreille, la poitrine, les muscles, la respiration et le souffle. «Le mécanisme vocal implique l'action coordonnée de nombreux muscles, organes et autres éléments dans l'abdomen, la poitrine, la gorge et la tête. En effet, c'est presque le corps entier qui influence le son de la voix, directement ou indirectement $\gg$ (Sataloff, 1992, p. 108). De plus, l'ouie et la voix sont reliées par le retour auditif et par la résonance physique, l'expérience immédiate de notre présence à travers la caisse de résonance de la poitrine et de la tête, la sensation de réverbération du son, principalement notre propre 
voix. En créant un monde d'espace et de temps fait de durée et de mouvement simultanément à l'avant et à l'arrière, en haut et en bas, à droite et à gauche, un alignement imprègne tout le corps, fixe ou en mouvement. C'est la raison pour laquelle l'audition et l'expression vocale relient les sensations ressenties du son et de l'équilibre à celles de la présence physique et émotionnelle.

Cette position problématise l'argument de Abu-Lughod et Lutz selon lequel « l'émotion peut être étudiée dans son caractère incorporé uniquement après qu'on a pleinement accepté sa dimension sociale et culturelle - discursive » (1990, p. 13). Bien qu'elles assurent qu'« en tant que produits culturels, [les émotions] sont reproduites dans les individus sous la forme d'expérience incorporée » (1990, p. 12), il semble peu raisonnable de soustraire le discours, ou la production et la circulation de sujets à travers les styles et les genres de discours, de la voix incorporée, de l'endroit de l'articulation verbale, du lieu de résonance du discours, en tant qu'habitudes entièrement émotionnelles. Les émotions sont peut-être créées dans le discours, mais cette création sociale est dépendante de la performance, qui naît toujours à travers des voix incorporées (voir Urban, 1991, p. 148-171).

Acoustémologie, acousteme : j'ajoute ces termes au vocabulaire des études sensorielles et sonores pour insister sur le potentiel du savoir acoustique, du son comme condition du savoir et pour savoir, de la présence et conscience soniques comme des forces puissantes qui façonnent la manière dont les gens donnent sens à leurs expériences. L'acoustémologie signifie une exploration des sensibilités soniques, plus particulièrement des modalités par lesquelles le son est essentiel à la fabrication du sens, au savoir, à une vérité de nature empirique. Cela semble particulièrement pertinent pour comprendre le jeu entre le son et l'équilibre que l'on ressent dans le sens et la sensualité d'une localisation, de la fabrication du lieu. En effet, les lieux ont potentiellement autant la capacité de réverbérer que celle de refléter, et les expériences et souvenirs incorporés que l'on en a peuvent résulter de manière significative du jeu entre cette résonance et cette réflectivité.

L'acoustémologie signifie qu'en tant qu'espace-temps sensuel l'expérience du lieu peut potentiellement toujours être ancrée dans une dimension acoustique. Il en est ainsi car l'espace régit la distribution des sons, et que le temps répartit le mouvement des sons. Cependant, le temps acoustique est toujours spatialisé ; les sons sont ressentis comme reliant des points en haut et en bas, à l'intérieur et à l'extérieur, en écho et en réverbération, en point-source et sa diffusion. Et l'espace acoustique est de la même manière réparti dans le temps; on entend les sons bouger, se localiser, placer des points dans le temps. Le positionnement du temps auditif est l'enveloppe sonique créée à partir des couches d'attaque, de suspension, d'affaiblissement et de résonance des sons. Le positionnement de l'espace auditif 
est la dispersion de la hauteur, de la profondeur et de l'orientation du son. L'espace-temps retentit inévitablement comme figure et fond, comme une venue et un départ. Sa présence est vers l'avant, vers l'arrière, côte à côte, et on l'entend selon des trajectoires d'ascension, descente, courbure, ou ondulation. Ce que ces formulations plutôt abstraites suggèrent simplement, c'est qu'expérimenter et connaître un lieu - le lieu ressenti, le lieu comme sensation - peut surgir à travers un jeu complexe entre l'auditif et le visuel, ainsi qu'à travers d'autres processus perceptifs inter-sensoriels.

\section{L'acoustémologie bosavi : corporéité commune de l'environnement, des sens et des arts}

À l'instar de leurs voisins de la forêt tropicale du Grand Plateau papou et dans la région environnante de la forêt tropicale de Papouasie-Nouvelle-Guinée, les Kaluli entendent beaucoup de choses qu'ils ne voient pas. La qualité diffuse du son est importante dans la forêt tropicale, et l'orientation du corps de ses habitants par le biais de l'ouie, l'écoute, et la voix a fait forte impression aux ethnographes qui ont travaillé dans la région (e.g. Feld, 1990 ; E. L. Schieffelin, 1976 ; Sørum, 1989 ; Weiner, 1991). Les Kaluli développent en général un sens de l'ouïe aiguisé pour s'orienter dans les lieux. Que celle-ci soit employée pour des activités forestières comme la chasse guidée par le son, ou prosaïques comme marcher le long de chemins forestiers ou prêter attention aux détails de la végétation environnante depuis une maison longue ${ }^{15} \mathrm{du}$ village, l'information localisée disponible à partir du son dans cet environnement excède souvent grandement celle qui est disponible par la vue, tant par la variété que par la prépondérance. Même si on remarque rapidement que l'ouïe est le sens le plus développé culturellement à Bosavi, l'audition est toujours dans un jeu avec les autres sens, particulièrement dans une dialectique étroite avec la vue. Cela tient au fait qu'une grande partie de la forêt est cachée à la vue, alors que le son ne peut pas être caché. Un Kaluli nommé Jubi me l'a un jour fait comprendre par analogie. Il disait que de même que les identités des danseurs costumés dans les cérémonies, ou celles des mediums agissant dans l'obscurité totale, sont révélées uniquement par la présence d'une voix chantante ou parlante, les présences de la forêt sont annoncées de manière sonore même quand elles sont cachées à la vue.

15. Le terme longhouse caractérise les grandes habitations collectives traditionnelles du peuple kaluli, qui prennent la forme de maisons longues ou allongées. Nous avons choisi de traduire ce terme par « maison longue ». 
Ainsi, la présence acoustique révélatrice est-elle toujours en tension avec la présence visuelle cachée dans les expériences primordiales de la forêt. Reliant l'expérience et l'expression, cette même tension s'applique dans les concepts poétiques kaluli - par exemple, le désir multi-sensoriel d'interpréter des chants, des conversations, des disputes, ou des histoires en « retournant », balema, leur surface pour révéler leur heg, « dessous », ou $s a$, « intérieur ». Les intérieurs et dessous retournés révèlent les profondeurs, significations, et subtilités sonores, et les implications des sons, poèmes chantés, discours allégoriques, ou costumes de danse, autant qu'ils révèlent les présences cachées des lieux forestiers - la signification des façons dont les lieux sont physiquement créés, comme par exemple la manière dont les pierres, les cascades, les montagnes ou les cours d'eau émergent comme des présences avec des passés « intérieurs » et « souterrains 》 riches de sens. Ainsi le lieu commun selon lequel les objets et les événements sont toujours plus que ce qu'ils paraissent, prend-il un caractère particulièrement sensuel et poétique quand il s'agit des modes kaluli d'interprétation des profondeurs et des dimensions de l'expérience locale.

Une autre manière dont la dialectique kaluli entre ce qui est caché et ce qui est révélé émerge est puissamment signalée dans le terme iconique, inter-sensoriel, mama, « réflexion » ou « réverbération ». Mama est l'image de soi-même dans l'eau ou dans le miroir ; c'est la réflexion en gros plan de soi-même dans le globe oculaire d'un autre, la présence visuelle du soi séparé du soi. C'est aussi le fragment auditif persistant d'un son qui s'affaiblit, sa projection vers l'extérieur tandis qu'il résonne en montant et s'évanouissant dans la forêt. Comme la clarté, en train de disparaître, d'une image dans le miroir, mama est la trace d'une mémoire auditive, de souvenirs sonores fragmentaires tandis qu'ils font écho. Et ane mama, une « réflexion-réverbération disparue », est un esprit, une absence humaine qui revient sous la forme d'une présence imaginée (souvent celle d'un oiseau). Annoncée par des éclairs visuels ou, de manière plus typique, par des sons qui attirent l'attention, ressentis sans être accompagnés d'une image visuelle correspondante, une présence de type ane mama stimule instantanément des souvenirs pleins d'émotions.

Ces jeux kaluli entre vision et son sont aussi en relation inter-sensorielle avec l'odeur de manière localisée. De nombreux exemples de la vie quotidienne pourraient être cités. Il est difficile d'imaginer le ruissellement d'un ruisseau peu profond au pied d'un palmier sagoutier sans sentir l'arôme de la moelle de sagoutier fraîche ou pourrissante ; l'expérience et le souvenir de la présence d'un lieu où poussent des sagoutiers sont profondément multi-sensoriels. De manière similaire, la sensualité dense de l'obscurité le soir, avec les voix se superposant aux légères pluies bruineuses et aux insectes et grenouilles de la végétation proche, est 
dans une continuité sensuelle avec les arômes fumés que les feux ou les torches de résine libèrent dans la maison longue et diffusent dehors dans l'air nocturne toujours humide. Évoquant le caractère diffus de ce sensorium cinétique, le même verbe bosavi, dabuma, ou absorption par l'oreille et le nez, comprend les processus d'audition et d'odeur. L'audition est la forme sans objet, la principale catégorie d'entrée ou d'absorption sensorielle ; l'odorat requiert d'indiquer le nom de l'odeur avant le verbe, de telle sorte que l'action de sentir charrie la sensation linguistique d'《entendre l'odeur ». Le potentiel métaphorique ici joue à l'inverse de l'idée occidentale synesthésique habituelle que les joies de la musique sont absorbées comme le « parfum de l'ouie » (Ackerman, 1990, p. 202).

Dans son acception la plus large, le caractère multi-sensoriel de l'acoustémologie bosavi est suggéré par la complexité des pratiques quotidiennes, reliant l'expérience sensorielle de la forêt tropicale aux processus artistiques à travers des moyens visuels, verbaux, musicaux et chorégraphiques. Ces pratiques sont comprises dans le discours par deux métaphores synesthésiques : dulugu ganalan, « son s'élevant par-dessus ${ }^{16}$ » et a:ba:lan, «écoulement ». Les deux sont importants dans l'expérience et l'expression de la localisation kaluli. Comme j'ai déjà traité de dulugu ganalan en détail (Feld, 1988), je vais brièvement rappeler ici son importance dans la relation entre les sens, puis concentrer mon propos sur l'écoulement. L'écoulement concerne le sens et la sensualité intriqués de l'eau qui s'écoule et relie les terres, ainsi que la voix qui s'écoule et relie le corps pensant, mouvant, sentant. Il concerne également l'emprise, l'étreinte persistante, du son et du chant poétique, la résonance de la voix dans la mémoire silencieuse. Ces notions d'écoulement se mêlent toutes dans la performance des cartes de sentiers qui sont un élément central des textes poétiques des chants.

« Le son qui s'élève par-dessus » est la construction métaphorique qui prescrit et décrit la forme naturelle des sons pour les Kaluli. Attirant l'attention sur l'axe spatial (《s'élève par-dessus ») comme sur l'axe temporel (《son ») de l'expérience, le terme évoque les manières dont tous les sons coexistent nécessairement dans des champs sonores antérieurs ou contigus. Quand elle est appliquée au monde sonore de la forêt tropicale, l'expression « le son qui s'élève par-dessus » met en lumière l'observation selon laquelle il n'y a pas de sons uniques et isolés à entendre. Tout est mêlé dans un soundscape intriqué. Les sons de la forêt changent constamment de figure et de fond pour créer des alternances et superpositions décalées, un sens du son qui est complètement intriqué et sans

16. L'expression lift-up over sounding est propre à S. Feld, et la traduction ne rend pas totalement l'aspect progressif et dynamique. 
délimitation. On n'entend pas d'unisson dans la nature. La présence et l'absence du son ou ses changements de direction et de dimension dessinent les coordonnées de l'espace en hauteur et en profondeur. Les sons interagissent constamment pour produire la sensation qu'un son va momentanément se détacher des autres, tout en traduisant en même temps l'idée que toute primauté est fluide, perdue aussi vite qu'elle est gagnée.

Dans la forêt tropicale, la hauteur et la profondeur du son sont facilement confondues. L'absence de signaux de profondeur visuelle se couple avec les ambiguïtés des différentes densités de végétation et avec les sons toujours présents comme le chuintement de l'eau, de sorte que la profondeur est souvent ressentie comme le caractère diffus de la hauteur bougeant vers l'extérieur, se dissipant en se déplaçant. « Le son qui s'élève par-dessus » code avec précision, quoique de manière suggestive, la sensation ambiguë que projette auditivement, kinesthésiquement et sensuellement un espace-temps : vers le haut est ressenti comme vers l'extérieur. Cette localisation du son est en même temps une sonorisation du lieu. On sait l'heure, la saison, et le placement dans l'espace physique à travers le panoramique sensuel du son dans la forêt. Cette manière d'entendre et de sentir le monde est intégrée comme un savoir du corps, une parcelle de l'« hexis » du corps au quotidien (Bourdieu, 1977, p. 87), le régime naturalisé de « techniques du corps » (Mauss, 1979) formant la base des rencontres habituelles des Kaluli dans leur monde.

Les Kaluli transforment ces rencontres quotidiennes avec des structures de type figure et fond acoustiques, étendant la naturalité de l'expérience du soundscape de la forêt tropicale à leur propre musique vocale et instrumentale. Les voix et les sons des hochets sont amenés à « s'élever par-dessus » comme les arbres de la canopée forestière ; les sons des percussions et des outils de travail sont faits pour «s'élever par-dessus » comme les cascades qui se jettent dans des bassins tourbillonnants. Ces idées sont développées par les Kaluli dans des pratiques musicales privilégiant un chant ou une sonorisation collective dense et étagée, qui évite toujours l'unisson. Pour créer un « son qui s'élève par-dessus », les voix, les instruments, ou les deux ensemble, doivent être synchrones tout en étant en décalage. Être synchrone signifie que le sentiment général est celui de l'ensemble, d'une coordination constamment cohésive dans le mouvement sonique et l'expérience participative. Cependant, les parties sont aussi en décalage, c'est-à-dire, en des points bien différents et mouvants du même cycle ou de la même structure de phrase, en concurrence même, à un certain degré de déplacement depuis un unisson hypothétique.

De plus, « le son qui s'élève par-dessus » est créé par le timbre, via une densification de la texture à travers un étagement d'attaques, d'extinctions et de 
fondus, d'accélérations, allongements et raccourcissements joueurs, de fission et de fusion des formes et des phrases sonores. Les parties musicales qui s'entrecroisent, alternent, ou se superposent créent une forme de participation qui estompe la distinction entre la concurrence et la coopération, reflétant la tendance kaluli plus générale à un égalitarisme rigoureux dans les activités sociales allant de la parole au travail, en passant par la négociation, le commerce et l'échange.

En phase avec ces dimensions de créativité musicale, les styles de peinture faciale reflètent au plan visuel le « son qui s'élève par-dessus » à travers un principe parallèle de type figure et fond, dans le contraste de texture entre le brillant et le terne, et dans le contraste de couleur entre le noir et le rouge. Les costumes de cérémonie vont plus loin et tirent parti de la densification des textures, en mélangeant de nombreux types de matériaux, en mariant et étageant la fourrure, les plumes d'oiseaux, les peintures rouges, noires et blanches, les coquillages, les bandes tissées, le bambou, les hochets, les serpentins en palmier, et les feuilles colorées. Tandis que le danseur de la cérémonie s'agite dans cet attirail, des couches de son « synchrone et décalé » émanent de ses coquillages et de ses serpentins en mouvement, « élevées au-dessus » par son tambour, son hochet, ou sa voix.

Englobant la nature, la musique, la peinture corporelle, les costumes, et les chorégraphies, « le son qui s'élève par-dessus » unit métaphoriquement l'environnement, les sens et les arts kaluli. De manière complémentaire, la notion d'a:ba:lan, « écoulement », infuse et unit également les expériences vécues du lieu à leur évocation expressive. Pour illustrer comment cela se produit, je m'intéresse en premier lieu aux manières ordinaires par lesquelles les Kaluli rencontrent, ressentent et nomment les lieux de leur monde, puis aux manières par lesquelles cet écoulement de la sensation du monde se transforme en poesis sensuelle du lieu.

\section{De la sensation à la dénomination : placer des sentiers d'écoulement dans l'expérience quotidienne kaluli}

L'importance du lieu et des noms de lieux dans l'expérience quotidienne, le discours et l'expression rituelle kaluli a été un sujet de longue date dans la recherche ethnographique et linguistique menée par mes collègues et moi-même au Bosavi depuis 1966. Les premiers travaux d'Edward L. Schieffelin reconnaissent la primauté de l'identification kaluli avec la localité :

L'identité de chaque communauté vivant dans une maison longue n'est pas associée de manière première avec l'appartenance 
clanique des gens qui habitent la a [maison longue]. Au contraire, au bout d'un certain temps la communauté s'attache à la région dans laquelle elle vit et vient à être connue par le nom de la localité. Ainsi, à titre d'exemple, les lignées de Gasumisi et Wabisi dont les maisons longues successives ont été situées dans le voisinage de la Crête Bagolo sont appelées le peuple Bagolo. (1976, p. 41)

En outre,

les noms de lieux, ceux du voisinage de la maison longue compris, font référence à des crêtes boisées, des rivières pleines de poissons, des lieux où sont les maisons et des lieux où poussent les sagoutiers où quelqu'un a vécu la majeure partie de sa vie [...] Ces lieux ont du sens parce qu'ils marquent le contexte de l'expérience passée. Les Kaluli s'identifient eux-mêmes avec les noms de lieux parce qu' ils voient leur propre reflet dans leurs terres. (E. L. Schieffelin, 1976, p. 44-45)

Dans son ethnographie, centrée sur le discours, de la socialisation kaluli (1979, 1986, 1990 ; Ochs \& Schieffelin, 1983, 1984), Bambi B. Schieffelin ne s'est pas concentrée de manière thématique sur le lieu, mais ses transcriptions des interactions familiales quotidiennes kaluli indiquent l'importance du lieu et du voyage comme sujets de conversation dans la circulation des discussions à propos de l'histoire familiale, des déplacements et du travail. Particulièrement intéressante est sa découverte de la manière dont le discours familial quotidien comprend une forme de discours rapporté dans lequel les noms de lieux sont précédés par un marqueur possessif à la troisième personne et suivi par un verbe d'état - par exemple, ene Bolekini sab, « il/elle est chez son Bolekini » (B. B. Schieffelin, communication personnelle, 1990). Cette forme relie habituellement le lieu à la personne, l'identité à la localité, et souligne la résonance affective des noms de lieux. Son importance dans les interactions entre l'enfant et la personne qui s'en occupe montre le sens biographique du lieu, que les enfants kaluli apprennent à s'approprier.

Mon propre travail sur la poétique kaluli (Feld, 1990) s'est concentré sur les manières dont la citation successive des noms de lieux dans les textes de chants et de lamentations improvise ou compose des cartes qui évoquent les souvenirs des événements, des périodes et des relations sociales. L'idée d'un tok, ou « sentier », est apparue comme l'un des dispositifs majeurs de la composition et de la performance d'un chant, et mes professeurs kaluli m'ont rendu bien conscient du fait que le pouvoir émotionnel et mémoriel des chants dépendait de 
leurs séquences de noms de lieux. La poursuite de ma recherche a révélé combien l'invocation de la notion de tok renvoie à un ensemble générique de suppositions à propos de la relation entre eux des lieux bosavi, et à travers cette relation, à une relation entre les gens, les expériences et les souvenirs. Tok signifie sentier, passage, canal, une entrée et une sortie sans direction, une ouverture dans le sens de route, piste ou chemin. Le lien tok implique des images multiples : une série de localités, de contiguités et de continuités d'espace délimité, une progression temporelle d'un lieu à un autre. Le concept permet ainsi d'ancrer la délimitation des lieux sous la forme de leurs relations.

Des tok sont régulièrement présents dans les expériences quotidiennes, alors que les Kaluli quittent et reviennent dans la zone où se situe leur maison longue, vont dans des jardins, des endroits à sagoutiers, ou rendent visite à d'autres communautés en maisons longues. Voyager dans le temps signifie toujours marcher sur des pistes, traverser dans le temps des lieux à la fois familiers et nouveaux, passer du temps avec les autres et du temps seul, traverser dans le temps les nombreux cours d'eau, ruisseaux et rivières qui découpent toutes les terres dans la forêt tropicale bosavi. Mais la vie kaluli implique aussi des activités quotidiennes dans la communauté de la maison longue même, et dans ses alentours : socialiser dans la maison longue, ramasser et couper le bois pour le feu, chercher de l'eau pour boire et cuisiner, fabriquer et réparer des sacs en filet, aiguiser des couteaux et des haches, fabriquer et réparer les vêtements, garder les cochons, faire des barrières, chasser et pêcher, couper, planter, semer, et s'occuper des jardins de bananes, pandanus, légumes, fruits et patate douce, et cuisiner, distribuer et partager la nourriture. Toutes ces activités rassemblent les Kaluli pour échanger et commercer, particulièrement la nourriture et la conversation.

En effet, on pourrait dire que la majeure partie de toute activité sociale kaluli est co-constituée dans l'action et la conversation, et on n'écoute pas les conversations Kaluli sans entendre parler de lieux. Des modes discursifs plus formels, incluant les histoires, les disputes, les négociations, les lamentations, et les chants, participent également à ce schéma, validant la centralité du lieu dans l'expérience de l'échange et de la mémoire. Au cœur de toute cette pratique de la parole se situent les pratiques de dénomination des lieux. Aux niveaux lexical et sémantique les plus basiques, ces pratiques indiquent l'importance perceptuelle dans la démarcation d'une géographie exceptionnellement variée, qu'on expérimente en s'engageant dans les continuités et discontinuités sensuelles de la forêt tropicale environnante.

Qu'il s'agisse d'un récit descriptif ou d'une instruction prescriptive, d'une conversation à propos de la maison, du monde immédiat, d'un voyage, ou d'un déplacement, chaque pratique de dénomination implique de créer un sentier à 
travers un co-référencement des noms de lieux spécifiques (désignés plus loin par $\mathrm{NL}$ ), avec une terminologie générique de formes de lieux. La distinction la plus fondamentale entre formes de lieux est celle entre hen, « terre » et ho:n, « eau ». Celles-ci sont nommées et évoquées par les termes hena:wi, « noms de terres », et ho:na:wi, « noms de cours d'eau ». Cependant, la distinction relie autant qu'elle distingue les dimensions du lieu, parce que l'expérience quotidienne au Bosavi comprend toujours une imbrication coordonnée des terres et des eaux que l'on nomme.

On retrouve bien cette coordination dans les subtilités de la plupart des noms génériques de types de lieux. À titre d'exemple, les deux types de formations terrestres les plus importantes sont fele et do:m. Le terme fele est en lien avec le mot $f e$, « cuisse », et fait référence à une étendue de terre relativement large et plate, qui tombe en s'affaissant d'un côté ou de l'autre - ce que les marcheurs australiens, dans un instructif contraste métaphorique, désignent comme une « selle » de terre. On atteint les fele, qui peuvent aussi faire référence à la surface relativement plane le long d'une crête, par une côte, et elles mènent à une pente de l'autre côté. Ces segments conjoints de côte, descente, et affaissement dans la terre sont ses «flancs », ou do:m. Les segments $d o: m$ impliquent toujours l'existence de fele au-dessus, en dessous, et/ou sur les côtés. Do:m a la même forme phonologique que le mot désignant « corps » en kaluli, et bien que cela puisse être fortuit, d'autres indices lexico-sémantiques et discursifs me portent à croire que l'image du corps comme « collines » ou « flancs » reliés par des « cuisses » est assez fondamentale pour les locuteurs kaluli.

En tous cas, fele et do: $m$ sont vécus comme des formations de terre en relation, et rarement de manière autonome. Ils sont inséparables de la présence aussi courante, mais beaucoup plus sensuelle, des cours d'eau. Marcher sur un do:m implique une étendue d'eau en-dessous ; une fois traversé, il y a un autre $d o: m$ à grimper de l'autre côté. Quant à fele, il implique un, ou généralement plusieurs, eleb d'eau gisant en-dessous sur l'un ou l'autre de ses côtés. Eleb renvoie, dans une élévation qui monte ou se courbe, au lieu où l'eau du ruisseau s'arrête. Les Kaluli paraphrasent ce phénomène comme la « tête » de l'eau et disent que l'eau « dort en descendant depuis sa tête ». En d'autres termes, l'eau s'incline, se déplace le long d'un corps gisant à terre, s'écoulant vers l'aval depuis sa «tête » légèrement élevée. Une autre paraphrase locale dit qu'à l'instar d'une personne qui se tient debout, l'eau s'arrête et s'oriente vers sa «tête ».

En fait, l'eau s'arrête en se déplaçant le long du do:m, vers le fele. En suivant l'expression locale, les guides kaluli sont capables de pointer que le eleb n'est pas sur le fele mais dans le do:m. C'est une autre manière de dire que le corps est semblable aux courbes de terre entre, autour et au-dessus desquelles l'eau 
s'écoule. Mais cette imagination incorporée va même plus loin, dans la mesure où, comme ces formes de terre principales sont reliées comme les cuisses au corps, le passage de l'eau à travers elles s'écoule comme le mouvement de la voix. La voix s'écoule par la résonance à travers le corps humain, reliant de manière sensible ses segments spatialement contigus, résonnant afin de relier sensuellement et renforcer le tout. De la même manière, quand l'eau s'écoule à travers la terre, elle est toujours reliée, toujours présente à titres multiples dans une variété de formes de terres relativement distinctes, contiguës, les mettant en lien et révélant leur unité.

Il convient de mentionner ici que mis à part certaines correspondances évidentes entre les formes désignées par hen, «terre », et ho:n, « eau », en kaluli et en français, il y a des difficultés considérables à commenter linguistiquement comme à paraphraser le sens de la distinction et de l'interconnexion de do:m, fele, et eleb en tant qu'images du corps ou du paysage. Des noms comme ces trois-là semblent, de manière inévitable, beaucoup plus abstraits en français que ce qu'ils doivent représenter pour les Kaluli, qui les expérimentent directement comme des signes d'une sensuelle évidence du lieu. Une partie de la difficulté à les saisir vient du manque flagrant de correspondance visuelle entre ces formes de la forêt tropicale bosavi et celles qui sont familières aux Occidentaux. Car tandis que les do:m sont relativement escarpés et pareils à une poitrine, et que les fele sont relativement plus plats et pareils à une cuisse, ces termes ne signifient pas vraiment « colline » ou « plaine », dans le sens qu'ont ces termes en français, pas plus qu'eleb ne signifie exactement la « source » ou la « fin »d'un petit ruisseau.

La difficulté réside également dans le fait que do:m, fele et eleb sont vécus et reconnus moins comme des formes purement visuelles que dans une appréhension multi-sensorielle, par la coordination de la marche, de la vue et de l'ouie - la kinesthésie et la sonesthésie ${ }^{17}$ d'un lieu que le corps en mouvement, ressentant et expérimentant, met en forme, rencontre et apprend à connaître. Entourés par une forêt dense, les Kaluli appréhendent les hauteurs, profondeurs, et densités autour de leurs pistes à travers des signaux que ressentent leurs pieds et leurs oreilles, tout autant, si ce n'est plus que, par des signaux visuels. Autrement dit, ils ressentent et entendent, au premier chef, si la terre devant eux est relativement plus plane ou plus escarpée que la terre derrière ou sur les côtés. La terre est presque tout le temps humide de pluie, donc la présence d'humidité dans l'air et la sensation glissante des différentes épaisseurs de boue sur les pieds sont

17. Ce terme nous semble avoir été forgé par l'auteur, aussi l'avons-nous conservé comme tel. 
essentielles pour s'orienter dans des lieux où la densité visuelle est importante. De plus, on peut entendre simultanément quels sont les types de présences de l'eau au-dessus, en-dessous, devant, derrière ou sur les côtés, et si ces cours d'eau diminuent ou augmentent dans leur présence. Cette sensualité de la localisation et de l'emplacement, de même que cette base corporelle, synesthésique et sonesthésique, de la connaissance, est au cœur d'une acoustémologie kaluli, une épistémologie sonore de l'emplacement.

\section{Lieux et localisation génériques}

Poursuivons avec les processus génériques : les noms relatifs à la terre et à l'eau prennent souvent la forme d'un nom de lieu spécifique auquel s'ajoute un qualificatif de description qui précise la forme du lieu (dorénavant, $\mathrm{NL}+\ldots$ _ $)$. Bien que le nom de lieu spécifique, ainsi que le qualificatif de description (comme nom abstrait), puissent figurer seuls, ils sont généralement combinés. Pour prendre les exemples les plus communs, on entend souvent les lieux cités ainsi : $\mathrm{NL}+d o: m$, ou NL + fele, ou NL + eleb. Dans le cadre discursif plus large, ces ensembles combinatoires peuvent être renforcés et éclairés par des particules de lieu et déictiques immédiatement accolés, dont certains portent des indications d'orientation spécifiques : en haut, en bas, au-dessus, en-dessous, derrière ou en face. Ces dernières, et d'autres marqueurs emphatiques et paralinguistiques, donnent des éléments sur la direction, l'élévation, la densité, et la profondeur de la terre ou de l'eau, ainsi que sur sa dimension vécue dans l'expérience - par exemple, si elle est ou non visible ou audible.

D'autres termes qui distinguent d'importants types de formations terrestres sont énoncés comme suit : NL $+d u g u$, pour la zone au pied d'une colline ou la partie la plus basse d'une colline ou d'une montagne ; NL + misiyo: pour un lieu en hauteur s'élevant depuis un do:m; $\mathrm{NL}+$ dagon, pour un site montagneux ou un sommet visuellement distinct; NL + kugun, pour une zone de forêt non exploitée, semblable à une vallée s'aplanissant près d'une colline ou montagne; NL + bulo, un segment de terre plat sur un fele. Par ailleurs, le même nom de lieu peut être associé à plusieurs modalisateurs descriptifs différents, pour montrer comment une large bande de terre peut se relier à une variété de formes spécifiques. Plusieurs lieux discontinus géographiquement sur le même terrain peuvent avoir la même désignation NL + forme, soulignant ainsi la complexité de considérer de manière abstraite la terre uniquement à travers sa dénomination.

Autres repères familiers dans la terminologie des lieux : les terres déboisées pour créer des espaces de vie. Celles-ci incluent $\mathrm{NL}+b a$ ou ba-daido: ; une clairière ou un espace défriché, comprenant une maison longue principale 
s'ouvrant sur une cour dégagée. Cette clairière est connectée à la forêt, aux jardins et aux endroits à sagoutiers, par des sentiers coutumiers de diverses tailles et usages, allant des pistes reliant les villages de trente mètres de large (gamane tok ou dalaka, de l'anglais track, pour les routes nationales), qui signalent des contacts renforcés et la présence gouvernementale dans la zone depuis les années 1960, au petit sentier défriché ou bourbeux ouvert dans la forêt, où les traces de pas des cochons ou des humains indiquent les déplacements quotidiens habituels. Ces types de pistes mènent au-delà des sphères de l'habitat, du jardinage ou de la famille, vers des lieux forestiers progressivement usa, « à l'intérieur », ou heno: usa, « à l'intérieur des terres ». Ils s'éloignent des villages et des chemins plus larges, et s'enfoncent profondément dans les forêts vers ilabode, « la végétation broussailleuse ».

Les lieux sont explicitement reliés en tant que sentiers par NL + tok, ou en associant un nom de lieu spécifique à un qualificatif de sentier comme soso:go:f, le point où deux chemins se rejoignent, localisant ainsi un lieu par son point d'entrée ou de sortie. Les discussions concernant les chemins indiquent aussi des qualités comme «droit » (digalo:), « courbé » (koagelo:), ou complètement « méandreux » ou se repliant sur soi-même (sisiali), de même qu'on peut les distinguer par les types de végétation ou de densité de la forêt.

De manière similaire, les noms de terres sont précisés par des marqueurs indiquant les impacts de la présence humaine. Les noms font référence aux sites de jardinage sur brûlis par NL + ikuwo: ou NL + egelo: (littéralement, « arbre-coupé » ou « plante-plantée »), ainsi qu'aux caractéristiques du jardin. Par exemple, NL + asak indique un bord de jardin avec une crête plantée au-dessus. NL + ikuwo: sak (《arbre-coupé » + asak) indique l'apparition d'une double crête, avec certains arbres coupés, d'autres s'élevant au-dessus ; d'où l'apparence d'une double canopée, l'étage du dessous repoussant sur une colline et l'étage du dessus comprenant la croissance d'origine sur la crête.

Les lieux où les sagoutiers poussent, sont abattus et transformés en féculent de base du régime alimentaire kaluli, relient encore davantage impact de l'homme, pistes au sol et cours d'eau, particulièrement les petits ruisseaux et bras d'eau. On nomme les endroits à sagoutiers ainsi : NL + ma:n kuwo: («sagoutier-coupé »), et le nom de lieu peut également caractériser la portion de terre ou le cours d'eau adjacent. Comme les noms des sites de maisons longues sont centraux dans la création de l'espace-temps unifié d'une communauté, la présence humaine est toujours en relation aux jardins et aux zones à sagoutiers portant un nom, et par là même essentielle à l'identité de celles et ceux qui travaillent et vivent à proximité. Les souvenirs - repas, travail, entraide - sont comme aimantés par ces noms, faisant du lieu un locus unifié de temps et d'espace. 


\section{L'eau}

Toujours entendue même quand elle n'est pas visible, l'eau a également une présence visuelle prodigieuse, une profondeur et une ampleur dans son voyage à travers les lieux, qu'elle relie et délimite à travers la forêt. La présence acoustique de l'eau change constamment selon les cycles saisonniers, gonflant et résonnant avec les pluies quotidiennes. Produisant un chuintement régulier à première écoute, l'eau revêt une variété de volumes et de distances sonores qui comprend de multiples présences subtilement différenciées. Ces dernières sont instantanément ressenties et interprétées par les Kaluli comme des indicateurs de la progression de l'espace-temps selon le jour et la saison. En plus des termes ho:n et eleb, de nombreux autres termes descriptifs renvoient à la variété des cours d'eau, dans leurs dimensions et leur localisation, tels qu'on les croise chaque jour. Ils incluent $\mathrm{NL}+$ kini, « courant vers l'aval »; NL + mogan, «bassin profond »; NL + bese, une « cavité », une dépression ou un petit bassin dans lequel l'eau ralentit ; et $\mathrm{NL}+$ sa: la « berge » d'un ruisseau ou d'une rivière, le bord de l'eau. Les cours d'eau sont de plus caractérisés en tant que sisiali, courbés, tortueux ou méandreux, avec des virages réguliers, ou tili, sans bassin, uniquement l'eau s'écoulant vers l'aval. Il y a aussi des formes d'eau particulières, comme ho: $n$-si, une petite source dont on puise de l'eau potable.

Les deux formes de cours d'eau les plus notables mis à part eleb sont celles qui relient les cours d'eau entre eux et délimitent des frontières significatives ou des changements d'élévation du sol. Pour les cours d'eau, $\mathrm{NL}+s o: k$ indique une « confluence », un endroit où les deux cours d'eau se rejoignent, et NL + sa indique une « cascade ». Parce que les confluences et les cascades fixent des démarcations qui ordonnent ensemble les formes de terre et d'eau, elles sont également importantes pour démarquer les zones de pêche, les frontières, et les droits. Les cascades elles-mêmes sont particulièrement remarquables tant pour les contours qu'elles inscrivent dans la terre qui les entoure, que pour leur impact dans la forme de l'eau en contrebas - par exemple, NL + sa-wel, « la crête de la cascade » ou son bord; NL + sa-mi, une « chute de cascade » ou le point de rupture des eaux; NL + sa-mogan, un «bassin profond » à la base de la cascade ; $\mathrm{NL}+s a-k o: f$, là où l'eau rompt un mogan sous la chute d'eau; et $\mathrm{NL}+s a-g a$, là où l'eau se réunifie après avoir rompu au-dessous de la chute.

Il existe d'autres manières par lesquelles les cours d'eau sont co-référencés avec des formes de terres, notamment $\mathrm{NL}+d a: l$, correspondant à une section d'eau qui s'écoule à partir d'un fossé déboisé ; NL $+d u$, caractérisant une terre ou une berge rocheuse entre deux segments d'un même cours d'eau ; et NL + min, quand un cours d'eau se sépare en deux puis se réunifie plus tard. Quand deux ruisseaux 
circulent à peu près en parallèle le long d'une longue bande de terre, coordonnant ainsi les caractéristiques de la terre et de l'eau, ils sont nommés ida:ni galiali, « deux d'entre eux s'étendant/restant ensemble ».

Mais la primauté sensuelle de l'eau émerge dans les pratiques de dénomination kaluli d'une autre manière, à travers la prééminence descriptive d'idéophones faits d'onomatopées pour le bruit et le mouvement de l'eau. Tandis que ces idéophones sont courants dans les conversations à propos des cours d'eau, ils sont seulement en de rares occasions des substituts pour des noms d'eau spécifiques ou des qualificatifs de l'eau. Six schémas emblématiques rendent les sensations sonores des mouvements de base de l'eau :

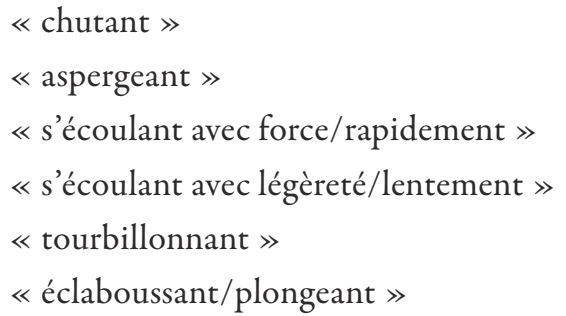

Des extensions supplémentaires de ces termes recourent à l'allongement des voyelles pour indiquer l'intensité, ou à la duplication des syllabes pour indiquer la continuité de durée. De plus, les idéophones peuvent être rattachés à une morphologie verbale classique dans la conversation, tout autant qu'à une morphologie aspectuelle spécifiquement poétique dans les chants. Dans quelques cas, les noms de lieux incorporent ces idéophones sonores. La cascade nommée Gulusa (le sa d'un cours d'eau nommé emblématiquement, le Gulu) est formée à partir de gulu $+s a$, « bruit d'eau tombant vers le bas $»+$ + cascade $»$. Bulusami, le lieu où le cours d'eau Bulu (là encore, le nom est idéophonique) rejoint la rivière Gamo en contrebas d'une grande cascade et d'un grand bassin, est formé à partir de Bulu $+s a-m i$, le ruisseau Bulu + 《le bord de la cascade », littéralement, le «son bruyant de l'eau qui roule vers l'aval »+ + le lieu de chute ». Dans ces exemples, les pouvoirs évocateurs de l'expression idéophonique placent la relation

\footnotetext{
18. Nous avons conservé l'orthographe choisie par S. Feld pour transcrire la langue Bosavi par souci d'exactitude, cf. note finale de l'article par l'auteur. Cependant, en français, on notera que le son orthographié « $\mathrm{u}$ » est à prononcer «ou », de manière à rendre la sensation sonore de l'eau qui s'écoule, par exemple « gululu » se prononcera « gouloulou ».
} 
directe du son et du sens dans la voix, reliant avec force l'expérience sensuelle quotidienne aux profondeurs esthétiques de la poésie.

\section{Terre, eau, terre entremêlées}

Dans son ouvrage The Sorrow of the Lonely and the Burning of the Dancers, Edward L. Schieffelin souligne la relation des terres et des eaux bosavi :

La plupart des lieux dans la forêt sont nommés d'après le cours d'eau qui donne à la terre ses contours dans ce voisinage [...] Les eaux, par leurs circonvolutions et leurs chutes, génèrent de nouvelles localités pour chaque nouvelle configuration de la terre. Le nom d'une localité porte, en effet, ses propres coordonnées géographiques, qui la placent dans une relation déterminée aux ruisseaux et cours d'eau qui s'écoulent à travers la forêt. (1976, p. 30)

De ce fait, l'expérience et la dénomination des terres et eaux bosavi s'interpénètrent toujours. L'association flexible entre des modalisateurs descriptifs de formes terrestres et des noms et des formes de cours d'eau spécifiques, et entre des modalisateurs descriptifs de formes d'eau, et des noms de terres spécifiques, montre cela avec le plus de force.

Des exemples privilégiés de ce processus proviennent des nombreux petits ruisseaux qui courent le long de larges bandes de terre. Dans la communauté que je connais le mieux, un ruisseau nommé Sulu n'est pas juste un cours d'eau abstrait, mais un cours dont le sentier se relie aux terres nommées Sulu do:mo: et Sulu fele. De plus, bien que Sululeb (Sulu + eleb) délimite l'endroit où le cours d'eau Sulu parvient à une source, c'est aussi un nom pour la terre en forme d'arche où le Sulu s'arrête. Et parce que la colline juste au-dessus de ce lieu particulier est l'endroit où les membres de Bono : ont déboisé la terre et construit leurs maisons longues dans les années 1960, 1970 et au début des années 1980, Sululeb est aussi le nom d'un site de maisons longues et, par extension, un point de référence fondamental pour les gens qui y vivent (Sululeb kalu, les gens de Sululeb). En même temps, tout le monde sait que Sulu est, de manière assez explicite, un ruisseau, avec des lieux portant des noms le long de ses rives, où il y a des variétés de sagoutiers et des noms de bassins (Sulu mogan), petits bassins (Sulu bese), de même qu'un courant vers l'aval (Sulu kini), une chute d'eau (Sulu sa, Sulu sa-wel), et une confluence (Sulu so:k). Ce que cet exemple montre est le jeu constant des résonances entre le particulier et le général, le personnel et le social, le temporaire et l'historique 
dont ces noms sont porteurs, ainsi que les relations espace-temps qu'ils établissent dans le lieu.

\section{Noms de lieux, références linguistiques et indétermination mémorielle}

La formule « NL + description de la forme » permet de nommer et, partant, de mettre en ouvre la localisation quotidienne, ancrant dans les conversations quotidiennes la description des lieux où l'on est, où l'on a été et où l'on va. En outre, les tendances dans la formation des noms de lieux recèlent un potentiel important en relation à la mémoire et à la biographie. Pour les Kaluli, comme pour leurs voisins Foi, « les noms de lieux agissent comme éléments mnémotechniques pour les actions historiques des êtres humains qui donnent leur singularité et leur sens aux lieux » (Weiner, 1991, p. 45). Certains noms de lieux jouent avec force la fonction de raccourcis, porteurs d'histoires au sujet d'événements historiques ou mythiques dont l'amplitude varie du commun au cosmique. Des lieux bosavi, par exemple, sont nommés d'après des origines mythiques ou des événements responsables de la création de tabous. D'autres sont directement construits comme des sources principales de pouvoir spirituel ou surnaturel, et des histoires sont attachées à ces noms de lieux pour indiquer pourquoi on évite de regarder ou de visiter le lieu, ou pourquoi on y évite certaines actions, mots ou comportements, parfois sous peine de susciter le ressentiment ou l'offense d'êtres mythiques et cosmiques. Certaines de ces préoccupations sont révélées dans des histoires bien connues ou assez ésotériques, de manière variable ; d'autres sont exposées dans les types d'échanges et de révélations propres aux séances de dialogue avec les esprits (E. L. Schieffelin \& Crittenden, 1991, p. 58-87 ; Schieffelin \& Kurita, 1988).

Au niveau le plus élémentaire, la structure du nom elle-même renvoie à diverses couches de références possibles. Un exemple très explicite de cette variété suit le schéma observé dans le nom de lieu Hinibululo:wo:, formé de bini + bulu + lo:wo: littéralement 《tremblement de terre $»+\ll$ fendu $\gg+$ nom, signifiant « un lieu où le tremblement de terre a fendu la terre ». Le lieu auquel on fait référence est une forme terrestre visuellement impressionnante et peu habituelle, et l'événement qui a créé cette dépression est rare dans l'histoire et à la marge de la mémoire historique actuelle. Comme le lieu en question se situe aujourd'hui le long d'une route gouvernementale principale, en dehors de la communauté de maisons longues de Suguniga, il y a un lien clair entre la sémantique de référence du nom de lieu, un événement, une forme terrestre située, et des récits importants dans l'histoire d'une communauté donnée et de ses membres. 
Cependant, des ensembles de noms de lieux chargés au plan sémantique comme celui-ci sont relativement rares dans la langue bosavi. Beaucoup plus présents, parmi les noms qui se distinguent par des références explicites, sont ceux considérés comme liés à un événement ou une action sans préciser la formation terrestre ou aquatique elle-même. Ceux-là sont assez importants dans l'histoire locale de la communauté. À titre d'exemple, tandis que je parcourais les terres Bono: en 1992, j’ai rencontré des lieux nommés Baoamisa:n et Baoho:mo:no: Le premier est formé de bao $+a+$ misa:n, signifiant « hutte de chasse rituelle »+ maison longue principale (《maison » + tête $»)$, c'est-à-dire, « site de maison longue où des gens vivaient pendant un bao a. » Le second, Baoho:mo:no: est formé à partir de bao + ho:n + mo:no, signifiant « cabane de chasse rituelle $\gg+\langle$ eau $\gg+$ <oisson $\gg$, indiquant un « lieu où l'on buvait pendant la tenue d'un bao $a \gg$.

Le bao a était une cabane rituelle et une période de réclusion, de chasse, d'enseignement ésotérique, et de liaisons homosexuelles entre hommes et jeunes garçons (E. L. Schieffelin, 1982). Cette institution s'est arrêtée en 1964 en raison de la menace simultanée sur la confidentialité de la pratique et de la demande de main-d'œuvre, suscités par l'arrivée des missionnaires pour construire la piste d'atterrissage bosavi. Les terres nommées Baoamisa:n et Baoho:mo:no sont proches d'un lieu nommé Wogole, le site du dernier bao a Bono:. Tandis qu'approche le moment (j'écris en 1995) où trente ans auront passé depuis la tenue du dernier bao a au Bosavi, et trente-cinq ans depuis la dernière cérémonie tenue en territoire Bono:, les significations et les récits liés à cette institution changent considérablement dans la population vivante. Des noms comme Baoamisa:n et Baoho:mo:no sont ainsi des rappels explicites des présences et activités passées avec lesquelles les membres de Bono: ou d'autres communautés ont des liens variables selon l'âge, le genre, et la connaissance des pratiques culturelles ésotériques. Les noms résonnent ainsi de manière variable, transparente ou profonde, et conduisent à des récits superficiels ou denses, de manière également variable.

Plus typiques encore sont les noms dont le potentiel de référence ouvre vers des récits biographiques ou historiques qui ont des résonances personnelles ou régionales variées et complexes. Par exemple, un matin de juillet 1992, Ayasilo et moi marchions sur la route gouvernementale devant l'ancien site de maisons longues de Bono: appelé Sululeb. Tandis que nous traversions le ruisseau Yolo, je lui demandai le nom de la colline s'élevant le long de la partie gauche de la route. Il répondit, d'un geste rapide, pointant du nez (une autre convention de localisation par le corps), que le lieu s'appelait Gasoamisa:n. On reconnaissait instantanément le nom formé à partir de Gaso $+a+$ misa:n, ce qui signifie Gaso, 
un nom d'homme, et « maison longue villageoise principale », ou le « lieu où Gaso construisit une grande maison longue de village ». Je demandai ensuite s'il y avait un « dessous », un heg ou une « raison » à ce nom, et alors que j'allumais mon magnétophone, Ayasilo fit remarquer (en kaluli, traduit ici de manière plutôt littérale) qu' « un homme nommé Gaso, le père du père de Wasoba, un homme du clan Wabisi, il y a bien longtemps, ayant quitté Muluma, vivant ici près du Yolo, il construisit une grande maison, plus tard la quitta, pour vivre à Nageba:da:n, c'est tout $\gg$.

En dépit de l'artifice du contexte (i.e., une sollicitation directe par question), il est frappant de remarquer la rapidité et le détail avec lesquels une personne et un événement mémorable de sa vie sont situés au plan narratif dans un espace-temps localisé. Gaso est rattaché de manière générationnelle à un homme âgé de la génération du père d'Ayasilo issu de leur communauté de maisons longues; il est aussi rattaché à un clan particulier, à un nom de terre d'une importance historique et actuelle centrale pour ce clan et la communauté environnante, au cours d'eau que nous étions alors en train de traverser, à un acte marquant, porteur de sens et d'audace pour la mémoire personnelle d'un homme, aux gens de l'ancienne communauté de maisons longues de Sululeb, et à une autre communauté de maisons longues impliquée dans une histoire de fission et de fusion avec celle d'Ayasilo. Des souvenirs biographiques, géographiques, historiques et régionaux sont ainsi rassemblés et révélés par le nom Gasoamisa:n, indiquant de manière réciproque comment une identité personnelle est localisée et comment les lieux sont essentiels à l'identité personnelle. Comme avec les Bedamini voisins, décrits par Sørum :

La temporalité est habituellement rendue concrète par la localisation, et les phénomènes temporels peuvent être vus comme objectivés à travers cette pratique. Le paysage est aussi de l'histoire. Le temps est localisé comme une partie immanente de la topographie. [...] Le temps et l'espace doivent être vus comme des dimensions d'une perception unifiée de l'espace-temps. (1989, p. 4)

Parfois, les structures des noms décrivent une formation terrestre uniquement. Dans ces cas, la signification du nom est transparente à un certain niveau, mais pas du tout évidente à d'autres niveaux. Par exemple, Walaheg est formée à partir de wala + heg, signifiant « falaise rocheuse $»+\langle$ en-dessous $\gg$. Rien ne fait allusion au fait que le lieu nommé ainsi est un surplomb de falaise porteuse d'un sens historique dans le do:mo Sulu, juste à côté de l'endroit où sa-wel Sulu, le rebord de la cascade Sulu, rejoint la rivière Sago. Mais en fait, de nombreuses histoires 
vont avec ce nom, au moins pour les membres de Bono:, qui savent que les os de certains de leurs proches sont dispersés sous la falaise à cet endroit. Quand je suis venu pour photographier la cascade Sulu - une des plus spectaculaires sur les terres Bolekini - Deina et Hasele m’ont emmené à Walaheg. Là, ils me parlèrent des temps où les os des morts de Bono: étaient déposés dans des falaises comme celle-là, pour que les cochons et les chiens ne les atteignent pas. Et notre présence ensemble sur le site a fait ressurgir d'autres histoires liées à ce contexte, comme celles concernant l'époque où les Bono: vivaient sur un site de maisons longues nommé Diwailo, et la façon dont, entre cette époque et celle où ils déménagèrent à Balasawel (on remonte dans le récit à partir des noms de maisons longues actuelles), les os étaient déposés à Walaheg. C'était la manière d'Hasele de localiser un espace-temps, expliquant que nous étions peut-être en train de contempler les restes du père de son père, un homme nommé Gulabia, ainsi que ses pairs, comme Tulunei, Hewabi, Sowiya:, Kogowa:, Diba:, et Mayowa.

Certains noms de lieux ont des structures étymologiques suggérant qu'une dimension référentielle ou un récit d'importance pourrait leur être attaché, mais un examen plus approfondi indique qu'elles sont largement transparentes. Un ruisseau nommé Haidokini pourrait certainement faire référence à un lieu en aval (-kini) d'un palmier haido, mais personne ne semble aujourd'hui attacher un sens particulier à cela. Et Momayo s'avère être simplement un nom de ruisseau, ba madale, « sans raison », sans lien de référence ou d'association à un lieu où les fleurs moma poussent. Des récits ou des significations ont pu être attachés à ces noms dans le passé mais sont désormais perdus. De manière similaire, les noms formés de NL + diko:lo, signifiant NL + «fabriqué » + anaphore, peuvent vouloir dire un « lieu de fabrication de $\mathrm{X}$ » ou un « lieu où $\mathrm{X}$ était fabriqué ». Mais interroger la présence d'histoires dans trois ou quatre termes de ce type suscita la plupart du temps des haussements d'épaules.

La remarque évidente à faire ici est la suivante : la signification d'un lieu ne commence ni ne finit avec la référentialité linguistique des noms de lieux. Le cœur vécu des noms et des pratiques de dénomination ne peut être réduit à la structure linguistique des toponymes ou à la variété des modalisateurs ou des marqueurs grammaticaux qui peuvent s'y attacher. Alors que certains termes semblent indiquer des significations publiquement accessibles, ces significations elles-mêmes ne sont en aucun cas fixées de manière prévisible, ni également ou largement connues. Qu'elles aient été retenues un temps ou longtemps, les significations peuvent être également contenues dans n'importe quel nom. Cela veut dire que chaque lieu et nom de lieu, quelle que soit sa formation linguistique et ses caractères distinctifs, peut évoquer quelque chose pour quelqu'un, et c'est selon un large spectre de possibilités que ces histoires sont partagées, 
significatives, pleines de sens, ou mémorables dans le temps pour des individus particuliers ou des groupes sociaux. Les significations de ces histoires dépassent largement celles qui peuvent être directement liées à la sémantique lexicale de la structure « NL + _ » et son contenu explicite. On pourrait suggérer comme une hiérarchie des types de noms de lieux, selon par exemple la charge affective qu'ils pourraient porter - noms de sites de maisons longues, noms de jardins et sites à sagoutiers, principaux ruisseaux traversés en rejoignant ces trois sortes de sites, lieux indiquant d'importantes relations aux proches et amis - mais en réalité, il y a une variation considérable des manières dont les noms portent et révèlent du sens. En fin de compte, ce sont les processus des activités vécues, incluant l'activité de parler, plutôt que la structure linguistique, la catégorie physique, ou la fonction, qui investissent les lieux d'une mémoire profonde, feuilletant le langage de vivant.

La sémantique des noms de lieux et les modalisateurs descriptifs qui peuvent leur être attachés est ainsi beaucoup plus indéterminée d'un point de vue référentiel que ne le sont d'autres domaines lexicaux. Parce qu'ils sont essentiels à la description et à l'expression d'expériences vécues, ces noms sont profondément liés à la sensation incorporée des lieux. Cependant, par-delà les références, les noms de lieux et leurs modalisateurs sont essentiels pour rendre la sensation, par la manière dont l'invocation verbale introduit l'espace dans une présence conceptuelle renforcée, que le lieu nommé soit simultanément vécu dans sa proximité physique ou pas. L'acte de nommer renforce le caractère naturel du lieu, le caractère tacite de ses dimensions ressenties en pensée et en action. Dans le langage de Heidegger :

La dénomination appelle. L'appel rapproche ce qu'il appelle. [...] Ainsi, il suscite la présence de ce qui était auparavant non appelé dans une proximité [...] Mais même de la sorte, l'appel n'arrache pas ce qu'il appelle de la distance, dans laquelle il est maintenu par l'appel même. L'appel appelle en lui-même, et par conséquent, toujours ici et là - ici dans la présence, là dans l'absence. (1971, p. 198-199)

L'art de nommer et l'art de raconter des Kaluli sont des pratiques quotidiennes de sociabilité remarquables, des formes de participation sociale profondément reliées à d'autres pratiques quotidiennes comme le voyage, le travail, les visites. En effet, les identités sociales, tout un imaginaire des relations des uns avec les autres, sont mises en acte au Bosavi, à travers la coordination de la conversation, du travail, des histoires, et d'autres activités de tous les jours, activités qui donnent une structure et une émotion à la fois sédimentées et renouvelées au sens du 
partage et de l'appartenance. Des couches d'expérience, depuis son propre lieu de naissance à d'autres lieux où on a vécu et qu'on a visités, font l'identité en cartographiant activement le lieu, reliant le passage du temps et la projection spatiale, réinscrivant le passé dans le présent, créant la biographie comme sentier.

\section{Chanter l'écoulement des sentiers : l'intensification poétique de la mémoire des lieux}

Écrivant à propos des pratiques de dénomination des lieux chez les Foi, voisins des Kaluli près du lac Kutubu, à quarante-cinq kilomètres au nord-est du Bosavi, James Weiner insiste sur le fait que «le langage et le lieu sont une unité. La manière dont l'action humaine et l'appropriation utilitaire s'inscrivent sur la terre est une iconographie des intentions humaines. Sa copie fidèle est le discours lui-même, qui, dans l'acte de nommer, transforme ces intentions en mémoires, fait d'elles une histoire-en-dialogue » (1991, p. 50). Le caractère entièrement naturel de ces intentions perpétuées fonde aussi une poétique kaluli du lieu. Cela signifie essentiellement que les dimensions de l'expérience que nous appelons « ordinaires » ou « esthétiques » sont mutuellement interdépendantes, qu'elles impliquent toujours des reconfigurations et des sensations naissantes. Le poétique est une figure d'émergence qui reconfigure un fond quotidien, renforçant à la fois ce fond et contribuant à sa solidité et sa fluidité. De même, le fond quotidien se tient prêt à être reconfiguré, à prendre une nouvelle hauteur ou une nouvelle profondeur, à évoquer plus qu'à signifier, à se mouvoir avec plus de force dans le domaine du sentiment et de la sensation. Ce jeu de type figure-et-fond est le mouvement central de la poétique des chants kaluli, et la transformation des noms dits en noms chantés intensifie profondément la sensualité de leur évocation.

La puissance et le plaisir esthétiques des chants kaluli émergent en grande partie de leur poétique textuelle des sentiers de noms de lieux. Composés et performés par des invités en contextes rituels pour susciter les larmes de leurs hôtes en évoquant le souvenir de personnes mortes et de lieux quittés, ces chants peuvent aussi être chantées au travail, dans les temps de loisir, et dans les activités quotidiennes par les femmes et les hommes tandis qu'ils traversent et passent du temps dans les lieux forestiers. Dans les contextes rituels comme quotidiens, les chants sont toujours réflexifs et contemplatifs, des qualités renforcées dans chaque cas par la construction d'une cartographie poétique, qui met en parallèle la création et la dénomination de sentiers et révèle ainsi à quel point les lieux laminent les souvenirs, les parcours de vie et les sentiments. Pour montrer certaines des cartographies imaginées et réalisées dans la poétique et les pratiques de performance chantée kaluli, je donne un bref aperçu des trois chants 
chantés par Ulahi de Bolekini, au proche ruisseau Wo:lu, qui sont enregistrés dans la partie «Se détendre au ruisseau » de Voices of the Rainforest [Voix de la forêt tropicale], un enregistrement du soundscape d'une journée dans la vie d'une communauté kaluli, en compact-disque et cassette (Feld, 1991).

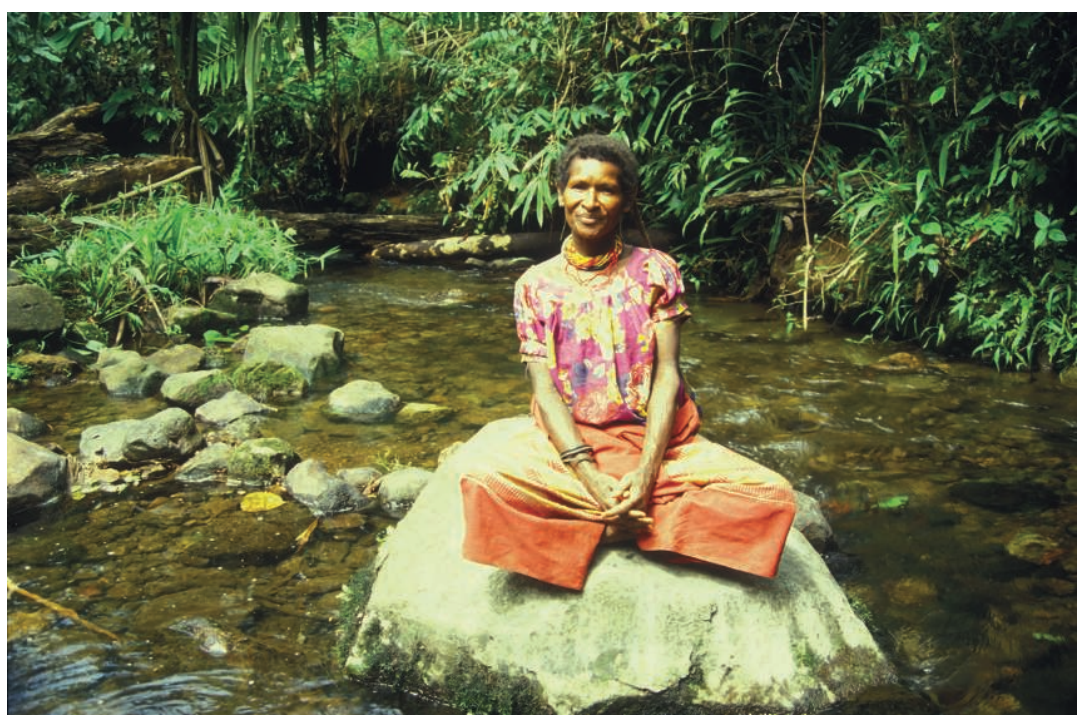

Figure 1

Ulahi chante au ruisseau Wo:lu

Photographie (c) Steven Feld.

\section{Cascades de chant}

Les trois chants d'Ulahi sont tous des versions courtes des genres kaluli les plus importants, gisalo, ko:luba, et heyalo. Ces trois genres sont mélodiquement, structurellement et historiquement distincts, mais leurs stratégies poétiques partagent les quatre pratiques conventionnelles kaluli textuelles et narratives appelées sa-salan, «parole intérieure », bale to, « mots retournés », go:no:to, « mots sons », et tok, « sentiers ». Sa-salan, « parole intérieure », consiste en des stratégies de reconfiguration du discours ordinaire de sorte qu'il n'implique que des contextes et situations de tristesse, de perte et d'abandon. Bale to, « mots retournés », ornent sa-salan à travers les techniques de la métaphore, de l'allégorie, et du voilement obscurcissant le sens. Go:no:to, « mots sons », comprend une large gamme de phonesthèmes, d'idéophones sonores. Et tok, « sentiers », implique une mise en narration de l'espace-temps du chant 
comme voyage à travers une progression de terres et eaux dénommées, associant l'imagerie de la citation et des affects à un ensemble particulier de lieux (à propos de la poétique kaluli, voir Feld, 1990, p. 130-162 ; à propos des cérémonies Kaluli et des genres de performance chantée, voir E. L. Schieffelin, 1976, p. 225-229).

Le chant gisalo d'Ulahi suit la structure en cinq parties typique du genre. Le chant commence avec une section appelée le mo:, ou « tronc », et talun, ou « vers ». Cette section consiste en des paires de segments. Le premier segment est un refrain, c'est-à-dire un texte et une mélodie qui se répètent à l'identique et régulièrement en alternance avec un couplet, un segment consistant en un texte changeant légèrement, chanté sur une seconde mélodie qui ne change pas. À chaque répétition du couplet, de petites portions du texte changent ; ces portions (transcrites plus bas en caractères gras) soulignent la manière dont la variation joue dynamiquement avec la répétition. Après plusieurs phrases de développement, la section entière « tronc + vers » est coupée poétiquement et mélodiquement du reste du chant. Cela est signalé par le sa-gulab, le « son $g u$ de la cascade », qui commence avec les deux vers « o » et «e $\gg$ entonnés en continu sur les hauteurs qui formeront le centre tonal du chant.

Après un bref développement textuel du sa-gulab, la prochaine section importante du chant est le dun, «branches », et talun, « vers ». Elle consiste en une nouvelle structure couplet-refrain, parallèle à celle que l'on trouve dans la section «tronc »du chant. De nouveau, des ensembles de segments appariés combinent un refrain répété et des couplets qui changent. La fin de la section « branches » du chant est appelée sa-sundab, le « nœud de la cascade » ou attache. Cela consiste en un ensemble répété et apparié de phrases poétiques et musicales menant au sa-gulu final, ou «bourdonnement gulu de la cascade », clôturant le chant, l'amenant jusqu'à sa fin sur son centre tonal par une énonciation prolongée des sons « o », « e » ou des deux.

La structure schématique suivante montre la conjonction de l'organisation poétique et musicale dans le chant gisalo d'Ulahi. La colonne à droite liste les hauteurs chantées, correspondant aux sections. Remarquez la manière dont l'inventaire des tons du chant se fusèle à mesure qu'il progresse, produisant l'image d'une cascade.

$$
\begin{array}{lll}
\text { mo: +talun } & {[(\mathrm{A}+\mathrm{B} 1)+(\mathrm{A}+\mathrm{B} 2)+(\mathrm{A}+\mathrm{B} 3)]} & \text { ९: mi-sol-la-do-ré } \\
\text { sa-gulab } & {[\mathrm{C}]} & \text { ९: mi-sol-la-do } \\
\text { dun }+ \text { talun } & {[(\mathrm{D}+\mathrm{E} 1)+(\mathrm{D}+\mathrm{E} 2)+(\mathrm{D}+\mathrm{E} 3)]} & \text { ९: mi-sol-la-do } \\
\text { sa-sundab } & {[\mathrm{F}+\mathrm{G}]} & \text { ९: mi-sol-la } \\
\text { sa-gulu } & {[\mathrm{H}]} & \text { ९: sol }
\end{array}
$$


Habituellement, les femmes ne chantent que des heyalo ou ko:luba courts quand elles travaillent ou se détendent. Ulahi, qui avait récemment réaménagé à Bolekini après un long séjour dans un autre endroit, m'a raconté que le processus plus long de penser à un gisalo lui vint à l'esprit tandis que son mari, Tulunei, et elle étaient en train de construire une nouvelle maison juste au-dessus de la colline Wa:feyo:. Son chant gisalo était encore en cours de composition quand elle le chanta de manière informelle au ruisseau Wo:lu. En raison de sa taille relativement courte, le chant d'Ulahi est une version musicale et poétique simplifiée du modèle gisalo complet de cérémonie, de trois manières. Tout d'abord, le chant omet une section mo:moso ou « tronc seulement » avant le mo: + talun. Un mo:moso consisterait, typiquement, en trois à six segments poétiques avant l'introduction du refrain en tant que tel. Deuxièmement, la chanson est simplifiée par le raccourcissement du mo: + talun, à trois paires au lieu des cinq à sept habituelles. Enfin, le raccourcissement de dun + talun, de trois paires au lieu de huit à dix, contribue encore à simplifier la structure. Une conséquence poétique de la brièveté du chant est qu'il y a relativement moins de lieux nommés que ce qu'on entend habituellement dans un chant de cérémonie gisalo. En outre, les lieux chantés par Ulahi tendent, en particulier dans le dun + talun, à être beaucoup plus éloignés qu'à l'habitude dans un tok de chant gisalo, qui chante souvent un ensemble de noms de plus en plus proches les uns des autres sur le territoire, ou bien alterne des lieux éloignés et très proches à mesure que le dun + talun se développe.

A

seyago

Wa:feyo:-sana selega siliki

we kidafi ganalabo-

weo:ba:mo: ganalabo-

we kidafi ganalabo-

B1

aowo: nilo : siyo :makaka :mi $0-O$

Wa:feyo:-sana gola

mesa:siyolo cassican à tête noire seyak (appelant)

perché sur un grand arbre sal sur la colline Wa:feyo

appelant dans un $u f$ [un autre gros arbre à bois dur]

appelant depuis le proche haido [un

palmier isolé]

appelant dans le uflà-bas

frère, je ne t'ai rien répondu

(appel triste en réponse à la colère du frère) je viens séjourner au bassin de la colline Wa:feyo: 
wo:funa: dok mesa:siyolo- viens rester au-dessus des feuilles du bord du ruisseau

Wa:feyo:-sana gola je viens rester au bassin de la colline mesa:siyolo Wa:feyo:

A

seyago

cassican à tête noire seyak (appelant)

Wa:feyo:-sana selega siliki perché sur un grand arbre sal sur la colline Wa:feyo

we kidafiganalaboweo:ba:mo: ganalabowe kidafiganalaboappelant dans un $u f$ appelant du proche haido appelant dans le $u f$ là-bas

B2

ada:yo: nilo: siyo:makaka:mi $0-0$

ada : je ne t'ai rien répondu

Diyo:so gola mesa:siyolo(appel triste en réponse à la colère de $a d a:$ ) ka:la:na: dok mesa :siyoloJe viens rester au bassin Diyo:so: Diyo:so gola mesa :siyoloje viens rester comme un poisson ka:la:n Je viens rester au bassin Diyo:so:

A

seyago Wa:feyo:-sana selega siliki cassican à tête noire seyak (appelant) perché sur un grand arbre sal sur la colline Wa:feyo

we kidafi ganalaboweo:ba:mo: ganalabowe kidafi ganalaboappelant dans un $u f$ appelant du proche haido appelant dans le uflà-bas

B3

aowo: nilo: siyo:makaka:mi frère, je ne t'ai rien répondu $0-0$

Elade gola mesa:siyolo(appel triste en réponse à la colère du frère) wo:funa: dok mesa:siyoloje viens rester au bassin Elade je viens rester au-dessus des feuilles du bord de ruisseau

Elade gola mesa:siyoloje viens rester au bassin Elade 
wo:funa: dok mesa:siyolo-

$$
\begin{aligned}
& \mathrm{C} \\
& o-o \\
& e-
\end{aligned}
$$

Kida:n-sagu "A:bo:lo:-"

Kida:n-sagu "A:bo:lo:-"

D

ni ka: wela:ligo:lo:

Kida:n-besewela:ligo:lo:

“Gayo" wela:ligo:lo:-e

E1

ne dogo misiyo:

nela-ba:ba:no:-

\section{A:ba:lebo:}

nela-ba:ba:no:- A:ba:

A:ba:leb a:ba:l kosa

dowabi(ki) ba:ba:-miyo:wo

e-

D

nika: wela:ligo:lo:-

Kida:n-bese wela:ligo:lo:-

“Gayo" wela:ligo:lo:-e

E2 je viens rester au-dessus des feuilles du bord de ruisseau

(eau tombant en cascade, son émergeant de la source)

(eau tombant en cascade, son réverbérant vers la source)

cascade Kida:n (appelant) « A:bo:lo » [nom de cochon]

cascade Kida:n (appelant) « A:bo:lo »

Je n'arrête pas d'appeler mon cochon n'arrête pas d'appeler là où le ruisseau Kida:n plonge n'arrête pas d'appeler « Gayo » [nom de cochon]

Je veux voir le sommet de la colline

le sommet de la colline au-dessus de la tête du ruisseau

Je suis venue voir le palmier noir solitaire se balançant au- dessus d'A:b:aleb (son réverbérant)

Je n'arrête pas d'appeler mon cochon n'arrête pas d'appeler là où le ruisseau Kida:n s'enfonce n'arrête pas d'appeler « Gayo »

dogo misiyo: nela-ba:ba:no:- Je veux voir le sommet de la colline 
So:lo:-sawa:lo:

nela-ba:ba:no:-

waya kosa dowabi(ki) ba:ba:- Je suis venue voir le palmier solitaire qui se miyo:wo

$\mathrm{D}$

ni ka: wela:ligo:lo:-

Kidan:n-bese wela:ligo:lo:-

"Gayo" wela:ligo:lo:-e

E3

kuguno: nela-ba:ba:no:-

Yagafo: nela-ba:ba:no:-

akol kosa dowabi(ki) ba:ba:miyo:wo

$\mathrm{F}$

gululu-lebe

So:lo:-sawa:l folebe

gululu-lebe

So:lo:-sawa:l folebe

G

mo fuwa:n-ibo

Kida:n sawa:l fuwa:n-ibe

mo fuwa:n-ibo

Kidan:n sawa:l fuwa:n-ibe

Je veux voir le bord de la cascade So :lo : balance

Je n'arrête pas d'appeler mon cochon n'arrête pas d'appeler là où le Kida:n plonge

n'arrête pas d'appeler « Gayo »

Je veux voir la vallée du ruisseau

Je veux voir Yagaf

Je suis venue voir l'arbre esprit solitaire akol

(sons de cascade continus) «gululu »

bord de la cascade So:lo: (déferle, faisant le son) «fo -»

(sons de cascade continus) «gululu» bord de la cascade So:lo: (déferle, faisant le son) « fo -»

eau (bruits continus d'aspersion)

«fuwa:n»

faisant le son «fu:wa:n» au bord de la cascade Kida:n

eau (bruits continusd'aspersion)

«fuwa:n»

faisant le son «fu:wa:n »enjaillissant à

Kida:n-sawa:l

$\mathrm{H}$

(son de cascade bourdonnant) 
Dans chaque segment A de la structure mo: + talun, le premier mot, seyago, annonce la présence d'un oiseau seyak ou sagelon, Cracticus cassicus, le cassican à tête noire. C'est un oiseau très sociable des bords de village partout présent au Bosavi, et dont les appels sonores, mélodieux et polyphoniques, sont entendus au petit matin et en fin d'après-midi. Commencer en chantant le nom de l'oiseau invoque sa présence ; les mots du chant sont ainsi compris comme des « mots sons d'oiseau » ou o:ba: go:no:to, annonçant que ce qui suit est énoncé depuis le point de vue d'un oiseau. Tandis que cette référence est directe et évidente ici, elle alterne ensuite entre arrière-plan et premier plan, quand la poésie passe d'un récit positionnant le chant comme la voix d'un oiseau, à un texte à propos d'un oiseau, une histoire de présence d'un esprit.

L'image initiale du lieu où se trouve l'oiseau est Wa:feyo:-sana. Dans la région natale d'Ulahi à l'est de Bosavi, sana est la variante dialectale Ologo du mot do:mo: donc Wa:feyo: sana est un pan de colline au-dessus du bassin Wa:feyo: sur le ruisseau Wo:lu. Les vers qui évoquent le fait de séjourner dans un arbre seleg, d'appeler depuis un arbre kidaf, et d'appeler depuis un arbre weo:b embellissent la profondeur de sana Wa:feyo, créant un sens de l'espace et du mouvement en cet endroit. Ces vers rendent l'auditeur ou auditrice perplexe, tout en attirant son attention vers ce qui suit en employant des variantes Ologo pour les arbres plus communément connus dans le dialecte local kaluli en tant que sal, uf, et haido. Les images cumulatives de deux arbres à bois dur solitaires se tenant sur une colline qui a été déboisée au-dessus du ruisseau Wo:lu et, de l'autre côté, d'un unique palmier demeurant seul tandis qu'une aire de jardinage est découpée tout autour, créent une triangulation entre la crête, la colline et le jardin autour des courbes du ruisseau. Ces vers donnent simultanément un certain sens de l'espace, du mouvement, de l'aire, du volume, de la profondeur et de la relation des lieux entre eux, suggérant des manières dont les vies et les histoires ont pu être écrites sur ces terres. Les lieux où un oiseau peut aller et les lieux depuis lesquels il appelle sont tous des marqueurs de la vie, du mouvement, de la concentration et de la dispersion des êtres humains.

Les segments B du mo: + talun commencent avec le terme de parenté « frère » + nilo: siyo:makaka:mi, qui veut dire : « je n'ai rien dit en retour pour te provoquer ou te mettre en colère $\gg$. C'est une phrase poétique classique. Elle dénote le sentiment d'innocence de quelqu'un sûr de son bon droit, comme pour dire, « je n'ai pas commencé ou alimenté ta colère ». Cela signifie que l'autre concerné se satisfait d'être injustement en colère et peu désireux de l'enterrer. Les Kaluli paraphrasent en général ce vers de la manière suivante : « tu m’as dit quelque chose mais je ne $t$ 'ai rien répondu en retour $»$. Ou bien, « je pense que je 
ne t'ai rien dit, mais tu es juste fâché (kulufeyab) contre moi ». Cette phrase vise à intensifier les états émotionnels au cœur du chant en tant qu'évocation : états de manque, d'abandon, ou sentiment de désolation, particulièrement celui causé par un refus, une colère ou une agitation injustes. L'emploi du son purement vocalique « o » comme vers poétique et mélodique entier à la suite de cette phrase initiale joue comme un connecteur, un soupir évocateur, un appel. Cela transforme non seulement les mots en « mots sons d'oiseaux » plus purement vocaux, mais créé aussi un espace-temps contemplatif pour l'auditeur, avec pour effet de souligner les mots qui viennent d'être dits, en les faisant réverbérer dans la mémoire.

Les trois lignes suivantes relient la colline Wa:feyo: au bassin du même nom, Wa:feyo: mogan (pour le terme Kaluli mogan, Ulahi emploie la variante du dialecte Ologo gola, tiré de l'idéophone sonore tourbillonnant golo ou gololo). Wo:fun est une espèce d'arbre touffu, épineux, poussant à une hauteur de quelques pieds seulement le long du ruisseau, au niveau de son bassin. Wo:funa: dok indique le point juste au-dessus de ces arbres nains de bord de ruisseau. L'oiseau dit qu'il vient séjourner au bassin sous la colline, qu'il vient vivre dans le feuillage de ces petits arbres près du ruisseau. Mais ce n'est pas un endroit où un oiseau peut vraiment rester, donc cela créé instantanément une image pathétique, celle d'un oiseau qui ne peut pas trouver de foyer, un oiseau qui se perd dans les profondeurs plus basses d'un cours d'eau au lieu de nicher dans les sommets des arbres au-dessus. Faisant suite à l'image précédente, dans laquelle un oiseau ne peut vivre avec son frère en colère, ces vers montrent clairement que l'oiseau n'a pas de foyer.

Dans les alternances des phrases B figurant en gras (B1, B2 et B3), il y a trois types de changements progressifs. Tout d'abord, il y a un changement dans le mot initial de la phrase, le terme de relation de parenté, de aowo:, « frère », à ada:, « grande sœur/petit frère », puis de nouveau à aowo:. Cette progression indique un changement vers, puis un retour depuis une forme intensifiée. Ada: un terme de relation réciproque pour grande sœur et petit frère, invoque la supplication et l'appel, portant l'idée que quelqu'un appelle de manière triste ; celui qu'on appelle devrait ressentir une profonde obligation à répondre. L'usage de ce terme implique une provocation stratégique, l'attente d'une action. Le retour du terme aowo:, « frère », implique que cette attente n'a pas été atteinte, qu'appeler ada: n'a reçu aucune réponse, n’a pas procuré à la sœur un réconfort ou une reconnaissance (au sujet d'ada:, voir B. B. Schieffelin, 1990, p. 112-35; Feld, 1990, p. 24-27).

Deuxièmement, il y a une progression de mogan Wa:feyo à deux autres bassins plus loin sur le ruisseau Wo:lu, Diyo:so gola (= mogan) en B2, et Elade gola 
en B3. Cette progression emporte le chant le long d'un cours d'eau qui quitte l'aire d'habitation du village Bolekini. Il y a un certain nombre de bassins dans le ruisseau Wo:lu, mais citer ces trois-là est particulièrement efficace. Wa:feyo: mogan est juste sous la colline où l'oiseau commence. Diyo:so mogan est le dernier bassin de Wo:lu directement sous les maisons où les Bolekini vivent. Et Elade mogan est juste au-dessus du lieu sur le Wo:lu où il est traversé par la grande route gouvernementale, signifiant avec clarté le carrefour vers d'autres terres.

Enfin, correspondant à ces changements des liens de parenté et des termes désignant les cours d'eau, il y a une progression, de l'image des feuilles au bord du ruisseau (wo:fun) au niveau du bassin, à celle d'un petit poisson à écailles qui s'y trouve (ka:la:n est une autre variante du dialecte Ologo, désignant un poisson similaire à l'espèce que les Kaluli appellent yan), et puis de nouveau à l'image des feuilles de bord de ruisseau wo:fun. Comprendre cette progression finale se rapporte davantage à l'imagerie du monde spirituel, où les bassins et les poissons indiquent la présence de ane mama, des « reflets disparus », esprits des morts. L'imagerie rend le schéma cumulatif et interactif des trois segments B assez explicite : l'oiseau ne peut vivre qu'avec des poissons dans les bassins, ne peut s'arrêter qu'auprès des arbres nains; à travers le sentiment d'abandon, quelqu'un a été réduit à l'état d'oiseau, réduit de fait à un « reflet disparu », une absence vivante transformée en présence d'un esprit.

Pris ensemble, les segments mo: + talun construisent une image narrative de départ et de rupture. Le matin, un oiseau vole au-dessus des terres Bolekini, mais quelqu'un est fâché par la présence de l'oiseau. Comme l'eau vive, l'oiseau quitte le village, en suivant le principal cours d'eau de Bolekini, sans lieu où s'arrêter sur le chemin, et arrive finalement à la lisière du village. Les caractéristiques de la terre, de l'eau, de l'arbre et du lieu sont associées à des images de perte, celles de la famille, des esprits oiseaux, des lieux quittés, des lieux où se rendre. Toutes ces images s'unissent avec force à travers le son et la présence d'une voix d'oiseau qui appelle dans une progression de verbes reliant discours direct et discours indirect.

\section{Sa-gulab}

Le $s a$-gulab brise ce récit et le reconfigure, tout d'abord à travers la pure musicalité sensuelle de ce son « en cascade », un bourdon sur le centre tonal intégrant la phonesthésie $\mathrm{du} \ll \mathrm{o} »$ qui se projette vers l'extérieur et $\mathrm{du}$ 《e $\gg$ qui réverbère. Cela est suivi d'un développement thématique essentiel dans le texte, exprimé par le vers répété Kida:n sagu, A:bo:lo:-. Ce vers réunit les images visuelles et sonores 
en juxtaposant un nom de cours d'eau à un phonesthème ${ }^{19}$ de description, puis à une voix qui appelle. La première partie du vers, les mots Kida:n sagu, mène l'auditeur à imaginer un lieu, une petite cascade du cours d'eau Kida:n où la chute d'eau produit le son $g u$. Cet endroit est relativement éloigné des lieux qui viennent d'être chantés dans le mo: + talun, mais tous les auditeurs kaluli sauraient que la « confluence », ou so:k, du Kida:n, est l'endroit où sa chute d'eau se déverse dans le ruisseau Wo:lu, considérablement en aval du mogan Elade. Ainsi le chant s'avance-t-il largement vers l'extérieur, créant l'attente que les « branches » du récit à suivre complètent l'histoire des lieux reliés au «tronc ».

La seconde partie du vers est juste « A:bo:lo: », un nom de cochon. Il n'y a pas de marqueur linguistique pour indiquer comment interpréter la juxtaposition du nom de cours d'eau et du nom du cochon ; rien ne vient souligner si le cochon se trouve en ce lieu, et appelle depuis ce lieu, ou s'il vit en ce lieu. Il n'y a pas non plus de marqueur linguistique pour indiquer qu'une voix appelle ce nom près de ce lieu. Les possibilités d'interprétation de la juxtaposition ne viennent pas de la sémantique linguistique, mais de la juxtaposition poétique du nom de cours d'eau et du nom de cochon, particulièrement via le jeu de leurs dimensions mélodiques et performatives.

Le contour mélodique du vers dans son intégralité est la clef de cette interprétation. La structure rythmique et mélodique est ici syllabique, ce qui signifie une hauteur et une pulsation par syllabe. Les hauteurs sont ascendantes pour les deux syllabes du mot Kida:n (notes la, do), puis descendent et se maintiennent sur les deux syllabes suivantes, le mot sagu (notes la, la). Les deux mots sont ainsi reliés de manière mélodique dans une arche (notes la-do-la-la) qui imite par le son la structure visuelle de la cascade dans la partie aiguë de la mélodie. Puis, après un temps de silence, la mélodie atteint sa hauteur la plus grave et de là, rebondit d'une tierce mineure pour se maintenir au centre tonal sur les deux dernières syllabes du nom du cochon A:b:o:lo (notes mi-sol-sol). Ces notes graves sont une icône sonore de la voix humaine, au registre plus grave ; le fait qu'il s'agisse d'une voix appelant le cochon est marqué de manière performative par l'allongement de la dernière syllabe. Cette juxtaposition textuelle et sonique de deux sortes d'appels continus, celui des cascades se précipitant (notes plus aiguës, la, do) et celui d'une voix appelant (notes plus graves $\mathrm{mi}$, sol), est ainsi marquée de façon imagée dans l'ordonnancement interne et la création de motifs combinatoires à partir du matériau musical. Cette relation se poursuit dans le

19. Terme créé par le linguiste britannique John Rupert Firth en 1930, pour désigner l'association entre un son et une signification dans une langue donnée. 
reste du chant, jusqu'à ce que l'appel de l'eau et celui de la voix languissante se mêlent pour finir par ne faire qu'un.

\section{Dun + talun}

Le $d u n+$ talun inclue trois ensembles appariés de type refrain (D) et couplet (E1, E2, E3). Le refrain (un texte et une mélodie se répétant à l'identique) est campé par une voix plaintive à la première personne. La voix appelle un cochon, et la phrase est marquée de façon aspectuelle pour transmettre l'idée selon laquelle « je n'arrête pas d'appeler ». Le temps et la continuité fusionnent ainsi avec le mouvement et l'espace, qui sont signifiés par la mise en relation directionnelle du nom de lieu Kida:n bese avec les lieux précédemment nommés. Enfin, au dernier vers, est ajouté le nom d'un autre cochon qu'on appelle. Quand les femmes se rendent sur les chemins en fin d'après-midi pour appeler leurs porcelets, elles marchent en général le long des cours d'eau. La différence ici est qu'à présent, les cochons sont appelés par un oiseau qui se déplace au bord du Wo:lu et du Kida:n. Combinant ce contenu avec le précédent changement de « frère » à ada: et de nouveau à « frère », il est désormais évident que la voix d'oiseau du chant est une voix féminine.

Les trois couplets en alternance E1, E2 et E3 (même mélodie mais le texte change : les variations paradigmatiques sont indiquées en gras) nomment, dans des vers successifs, un relief, un nom de lieu correspondant, et un arbre qu'on y trouve. Les premiers vers progressent en nommant un sommet de montagne (E1), un autre sommet de montagne (E2), et une vallée (E3). Les seconds vers nous renseignent sur des noms de lieux réels mais pas précisément sur les types de formations de terre identifiées dans les premiers vers, provoquant ainsi de la surprise et renforçant l'impact poétique. A:ba:leb (A:ba + eleb) n’est pas une montagne mais la tête d'un ruisseau, So:lo:-sawel n'est pas un sommet de montagne mais une crête de cascade, et Yagaf n'est pas exactement une vallée mais un flanc de colline pentu. Citer la forme du lieu avant le lieu particulier nommé crée l'espace-temps d'un déplacement vers ce lieu. Les troisièmes vers nomment tous des arbres situés près du lieu indiqué dans le vers précédent ; d'abord, deux palmiers isolés, a:ba:l et waya, puis un arbre esprit akol, immense et arc-bouté.

La coordination spatiale et temporelle du vol de l'oiseau se déplace le long du sentier tracé par ces trois ensembles parallèles de segments de vers. Cette immersion spatio-temporelle est de plus soulignée par les constructions verbales. Les deux premiers vers de chaque couplet finissent par la forme verbale au futur et à la première personne, « je verrai », souvent paraphrasée par les Kaluli par « je veux voir ». Le troisième vers de chaque couplet approfondit le sentiment 
du passage du temps, en terminant par la forme passée accomplie ba:ba:miyo:wo: « est venu et a vu ». Cet effet est encore compliqué par kosa dowabi(ki) qui le précède immédiatement, un aspect inaccompli indiquant que l'arbre est balayé continuellement par le vent. Ces vers progressent donc d'une image de l'oiseau visitant les arbres le long de son chemin, « venant pour voir », à celle d'un oiseau qui « est venu et a vu ».

Le pathos contenu dans cette imagerie est renforcé par le fait que les arbres dans les deux premiers couplets (E1, E2) sont de grands palmiers solitaires, et non des arbres qui peuvent être des foyers pour l'oiseau. Seul l'akol nommé dans le dernier vers de E3 - qui est aussi le dernier vers de tout le dun + talun - est le siège d'un arbre esprit, un arbre dont les fruits sont de la nourriture pour un oiseau esprit. Dans cette section la poesis repose sur le sens de l'écoute du discours rapporté de l'oiseau. Le discours est, bien sûr, un bale to, « des mots retournés » au plan métaphorique, parce que les arbres sont le frère de l'oiseau. Ces variations «veut voir/verra » et « est venu voir » indiquent que, parallèlement à son tronc, les branches du chant continuent l'histoire de la perte, du mépris d'un frère et de la tristesse de la chanteuse. Cela est renforcé par la dernière image de lieu, celle d'une descente vers Yagaf et la présence d'un arbre esprit akol solitaire et très rare à cet endroit.

\section{Sa-sundab + Sa-gulu}

L'imagerie de sa-sundab reconfigure fortement ces branches (comme la chute d'eau précédente le fit pour le tronc du chant) en recourant à des ressources poétiques non narratives. Le sa-sundab évoque trois sortes de bruits d'eau : les sons de chute et de bourdonnement gululu des immenses chutes de So:lo: et Kida:n ; les sons fo de leurs eaux s'agitant et jaillissant contre les rochers ; et les sons fuwa:n des eaux se diffusant en gouttelettes et restant sur place. Ces idéophones fusionnent la sensation de l'eau tombant de haut en bas, surgissant depuis le bassin en bas, s'écoulant au loin, et se répandant à la fois sur les rochers et sur la surface de l'eau même. Créant directement le sentiment spatial de la présence de la cascade, cette phonesthésie de l'écoulement évoque également le temps, à travers la sensation de la connexion et du mouvement de l'eau. En plus de fusionner cet espace-temps sensoriel, la section sa-sundab clôt le chant avec une mise en parallèle située dans l'espace, reliant les deux principaux cours d'eau, le So:lo: et le Kida:n, qui coulent en parallèle, ida:ni galiali, pour rejoindre le Wo:lu, définissant de manière décisive d'importantes connexions pour le peuple Bono: vivant à Bolekini. Le chant se termine avec un unique son de bourdonnement de sa-gulu, le « o » coulant, ouvert, emportant le chant avec l'eau, tandis que la voix d'Ulahi diminue. 




Figure 2

Le ruisseau Gulu ; l'eau s'écoule à travers la terre comme la voix s'écoule à travers le corps.

Photographie (c) Steven Feld.

\section{Poétique du lieu}

Le chant d'Ulahi coordonne les lieux nommés avec des arbres, des qualités de la lumière et du vent, des sons, et avec des actes et des paroles qui émeuvent. Ulahi crée des images d'un oiseau suivant un cours d'eau, des images de départ, de rupture familiale. Le tok du chant, son sentier, montre une interpénétration des noms de terre et d'eau, tous reliés aux ressources poétiques de la « parole intérieure », des «mots retournés » et des «mots sons ». Cependant, comparé à la plupart des chants gisalo, particulièrement ceux performés pendant une cérémonie gisalo, le chant d'Ulahi ne nomme pas beaucoup de lieux. Son pouvoir d'évocation ne dépend pas de la quantité ni des détails portant sur les lieux nommés, mais de leur caractère inter-relié, de la mesure avec laquelle ils cartographient l'histoire d'un lieu qui résonne émotionnellement avec la conscience de soi personnelle, biographique et historique pour des auditeurs Bolekini. À travers le chant, un auditeur Kaluli est suspendu dans les lieux, il passe à côté et à travers eux, fait un grand tour intérieur dans le micro espace-temps intériorisé de l'écoute. La dénomination du sentier créé un cadre pour mettre en images des ensembles de relations situées dans l'espace, antérieures, contiguës ou continues, mais pas nécessairement linéaires. Le chant d'Ulahi, qui naît à mesure qu'il se fait, pourrait finir avec presque deux fois plus de lieux et de détails qu'il n’en contient. Mais la stratégie narrative mène les auditeurs kaluli à imaginer un 
sentier de connexions importantes, créant un tok riche et cohérent, un sentier évoquant les jeux croisés de la présence et de l'absence à Bolekini.

Ces pratiques poétiques s'articulent puissamment avec la proposition générale de Keith Basso selon laquelle :

On peut penser que les noms de lieux sont parmi les symboles linguistiques les plus chargés et les plus évocateurs. En raison de leur lien inséparable aux localités particulières, les noms de lieux peuvent être employés pour convoquer une immense gamme d'associations mentales et émotionnelles - associations du temps et de l'espace, de l'histoire et des événements, des personnes et des activités sociales, de soi et des étapes dans sa vie [...] Les poètes et les compositeurs de chants ont longtemps compris que l'économie de l'expression peut renforcer la qualité et la force du discours esthétique, et que les noms de lieux sont prêts à être exploités à cette fin. (Basso, 1988, p. 103)

\section{Ulahi}

Comprendre l'évocation personnelle et sociale du chant d'Ulahi requiert de connaître davantage sa vie à Bolekini et la manière dont sa biographie colore ce que les auditeurs en général peuvent ressentir quand ils l'entendent chanter. Le trait le plus saillant de la position sociale d'Ulahi est qu'elle est une étrangère qui vient d'une région éloignée où les gens parlent le dialecte de la langue Bosavi de l'est, ou Ologo. Mariée dans la région centrale du dialecte kaluli en tant que seconde épouse d'un homme du clan Bono: appelé Tulunei, elle indique son extranéité linguistique par le fait qu'elle prononce son nom Sulunei.

La vie de femme mariée d'Ulahi a été dominée par les frasques de son mari, par son favoritisme envers sa première épouse, Eyo:bo, et sa longue histoire de conflits avec les hommes de son clan (au sujet d'Ulahi et de son histoire familiale, voir B. B. Schieffelin, 1990, p. 43-50). Tulunei et Ulahi sont à divers égards une étude comparée de tempéraments. La douceur quotidienne du comportement public effacé d'Ulahi correspond à la profondeur émotionnelle de ses chants contemplatifs et de sa voix expressive. Tulunei, à l'inverse, est un homme bruyant et ouvertement fantasque dont le tempérament est légendaire à Bolekini, comme l'est sa tendance rapide et irréfléchie aux démonstrations explosives de plainte, de demandes d'attention et de colère, qui se manifestent occasionnellement par le fait de frapper ses femmes, tout particulièrement Ulahi. Le fait qu'Ulahi ait cultivé des relations proches et distinctes avec Bambi B. Schieffelin et moi, travaillant 
comme une de nos assistantes régulières en transcription et en traduction depuis le milieu des années 1970, a été un prétexte pour des démonstrations occasionnelles de jalousie de la part de Tulunei. Ses tentatives de s'approprier les biens ou l'argent qu'Ulahi avait gagnés s'inscrivent dans sa tendance récurrente plus générale à être agité et à se sentir floué, qui est une marque familière de son discours devant les gens du clan Bono: qui ont vécu à Sululeb (un ancien site de maisons longues) et qui vivent actuellement à Bolekini. Dans le feu de disputes avec les hommes de son clan, Tulunei tend à agiter de manière dramatique et tranchante la menace d'emmener sa famille dans une autre communauté. Mais tôt ou tard, il se trouve toujours mêlé à une altercation dans le nouvel endroit et ramène sa famille à Bono: quelques années plus tard.

Quand le chant d'Ulahi fut enregistré en août 1990, la famille Tulunei avait récemment ré-emménagé dans le nouveau lieu Bono: à Bolekini. La famille était en train de construire une maison séparée au-dessus de la colline Wa:feyo:, de l'autre côté du ruisseau Wo:lu par rapport à la maison longue du village. Donc le chant d'Ulahi est né dans une période inconfortable, à un moment de retour dans un lieu où les relations portent un historique de tensions. En outre, chaque départ et chaque retour chez Tulunei sont entrés en résonance avec un sens de la perte et du déplacement propre à Ulahi, car c'était toujours elle qui partait pour calmer la colère des querelles conjugales. Le chant d'Ulahi reflète ces expériences et souvenirs d'inconfort liés au lieu, en évoquant poétiquement une carte de la marginalité, un sentier chanté au sujet de la familiarité d'un lieu qui n'est pas chez elle, à double titre - ce n'est ni le lieu d'où elle vient, ni le lieu où elle s'est installée sans le quitter, y retournant, puis le quittant, puis y retournant encore.

\section{Genrer l'espace et le temps du chant}

Le chant d'Ulahi ne porte pas seulement sur l'inconfort d'être dans et hors d'un lieu. Ses réflexions sont genrées, et ceci est une dimension unique et innovante de sa poésie. Elle réalise cette caractérisation de genre tout d'abord avec l'emploi du terme ada:. Dans les chants gisalo, le jeune frère invoque en général ada:, pour supplier une sœur plus âgée ou pour se plaindre de l'absence de réponse de cette dernière. Le « tronc » du chant d'Ulahi inverse l'appel à ada: de sorte que c'est la sœur qui appelle son frère. Une fois qu'il devient clair que c'est une voix féminine, elle renforce celle-ci dans les sections « cascade » et «branches » en introduisant le thème de la poursuite et de l'appel aux petits cochons, une activité quotidienne largement féminine.

Comprendre le gisalo d'Ulahi nécessite d'examiner sa présence genrée dans un espace d'absence. Les hommes n'ont pas organisé de cérémonie gisalo au 
Bosavi depuis 1984, et peu d'hommes Kaluli composent des chants gisalo. Cela est très significatif, car le gisalo est invoqué localement comme la plus Kaluli des formes d'expression Kaluli. Gisalo signifie de manière générique à la fois « chant » et « cérémonie », mais aussi le type de chant et de cérémonie (parmi cinq) que les Kaluli considèrent comme le plus complexe et le plus émouvant. C'est aussi le seul dont les Kaluli revendiquent l'origine. Le gisalo fut un jour le projecteur culturel le plus vif porté sur les hommes de Bosavi et leur intérêt pour la provocation et la maîtrise. Il privilégiait tous les règnes de l'action masculine, les hommes en mobilisant d'autres, les exhortant par le chant à réagir, cultivant des sphères d'influence, trouvant épouses, et impressionnant les autres à travers la provocation dramaturgique et la persuasion poétique. Le gisalo fut bien un jour le sommet incontestable de l'action esthétique et rhétorique au Bosavi, et comme l'étude ethnographique d'Edward L. Schieffelin (1976) le montre, c'était un spectacle par essence masculin.

De nos jours, cependant, la plupart des jeunes hommes kaluli sont plus actifs dans d'autres domaines : ils manient de l'argent, établissent des commerces, développent leurs talents rhétoriques dans des cours de catéchisme évangéliques, et obtiennent des contrats pour travailler à l'extérieur sur des projets d'extraction de pétrole et de bois. Pour certains hommes impliqués dans ces domaines, ou pour d'autres plus satisfaits de voir cela depuis la périphérie, l'attrait du cérémonial a disparu avec leur conversion au christianisme, un effet de la présence de missionnaires évangélistes depuis le début des années 1970. D’autres pressions induites par la création d'une économie davantage fondée sur l'argent et les exigences financières grandissantes du système de dot fournissent aux jeunes hommes kaluli de nouveaux contextes et débouchés pour l'évocation, la maîtrise et la démonstration d'énergie. La participation aux cérémonies n'est plus importante pour cultiver les talents rhétoriques et dramaturgiques qui étaient si essentiels au style masculin de présentation. Composer et chanter des chants gisalo est désormais une activité que la plupart des hommes kaluli associent avec la contribution que les hommes non chrétiens peuvent apporter aux cérémonies locales le jour de l'Indépendance de la Papouasie-Nouvelle-Guinée. Ainsi l'importance du cérémonial dans la trajectoire des hommes, dont le gisalo fut un jour l'apogée, a-t-elle complètement décliné depuis le début des années 1980.

Pendant ce temps, les femmes bosavi, par tradition, limitaient presque entièrement leur pratique de composition et de chant au heyalo, un genre importé et relativement mineur, bien que certaines, particulièrement les femmes de l'est du Bosavi, chantaient aussi dans le style ko:luba. Suivant cette tendance, Ulahi a composé et chanté de nombreux chants heyalo et ko:luba; ce n'est que récemment qu'elle a développé un intérêt pour la composition gisalo. Cet intérêt 
est clairement inhabituel, et même extraordinaire, et au moment où Ulahi entama son chant au ruisseau Wo:lu, ma mâchoire se décrocha presque de surprise. Ma réaction fut loin d'être unique. Les « ah ! » de réaction les plus étonnés, que faisaient et font toujours les hommes et les femmes kaluli en écoutant Les voix de la forêt tropicale [Voices of the Rainforest], arrivent toujours juste au moment où ils entendent Ulahi chanter les premières phrases de ce chant gisalo.

De manière habituelle et prévisible, les femmes à Bolekini furent très favorables au chant, réagissant en général aux premières écoutes par une formule : mada ko:le sele, « vraiment très différent », fort enthousiaste. Mais les hommes du Bolekini furent suspicieux à l'encontre de ce chant, qui les rendait un peu anxieux. Ils me rappelaient tous que le gisalo est chanté par des hommes, et certains me demandaient en privé ou en public pourquoi j'incluais une femme chantant le gisalo sur la cassette. Je répondais qu'il n'y avait pas d'hommes qui composaient le gisalo. Ils haussaient les épaules et disaient des choses comme : Hede ko:sega..., «c'est vrai, mais... », leurs voix s'estompant jusqu'au silence. Hasele concéda que le bale to, les « mots retournés » du gisalo d'Ulahi, étaient halaido, « durs », en d'autres termes, fortement évocateurs. Mais comme le chant n'était pas chanté par un homme pour une cérémonie dans le but de provoquer les larmes, ou par un medium pour une séance au cours de laquelle il aurait annoncé la présence d'un esprit particulier, il conclut que cela était defe sa:lan, plus ou moins un « discours illustratif », signifiant que ça n'était qu'un exemple, quelque chose montrant la nature du gisalo sans en être un véritable. Dans le fond, cela revenait à reconnaître que le chant d'Ulahi était une forme nouvelle et différente de gisalo, un chant d'expression personnelle plutôt que de provocation sociale. L'histoire que Hasele créa pour les autres hommes et lui-même était que ce chant était composé pour expliquer aux Américains ce qu'était un gisalo. Certains estimèrent qu'il s'agissait là d'une explication parfaitement valable, et la firent leur.

$\mathrm{Qu}$ 'Ulahi riposte explicitement, par sa parole, contre ses problèmes de vie commune avec Tulunei et la difficulté à trouver un endroit pour vivre, ou pas, son chant est une incursion féminine puissante dans un espace d'expression masculin momentanément inoccupé. Néanmoins, même si Ulahi compose et chante dans un genre marqué comme masculin, le chant et son matériau poétique sont dans une certaine continuité avec ses pratiques de composition heyalo et ko:luba des vingt années précédentes. Quand je l'interrogeais à propos de son chant, Ulahi elle-même revenait souvent à une expression familière. Elle disait que c'était un gisaloba madale, un gisalo « sans raison », ou « sans but ». C'est une phrase compliquée à comprendre, mais j'ai entendu Ulahi et d'autres chanteurs l'employer auparavant en référence à des compositions de nature réflexive. Tandis que ba madale peut vouloir dire « sans fondement » et être une caractérisation 
négative, particulièrement quand il modifie un verbe de parole, cela peut aussi signifier « juste par soi-même » ou « juste pour soi-même ». Et c'est ce qu'Ulahi soulignait auprès de moi - le fait que son gisalo était juste pour elle-même : Nina:li asula:sa:ga:ka sa-molan, «y ayant pensé seule, j'ai pu le composer. »Un ba madalegisalo signifie que le chant n'émanait pas du projet de provoquer les larmes chez une personne en particulier, comme un chant de cérémonie pourrait le faire. Au lieu d'avoir un objectif d'évocation particulier, dirigé vers un autre, le chant d'Ulahi était composé de manière réflexive, pour elle seulement. Plutôt que d'être un moyen de persuasion, son chant est né comme quelque chose à chanter seule, pour explorer ses sentiments à propos de son retour en terres Bono:, après un temps considérable passé ailleurs.

\section{Quels sont vos noms?}

Quelques moments après avoir fini son gisalo, Ulahi continua avec un second chant, une courte accroche, dans le genre ko:luba:

$\begin{array}{ll}\text { wo:wo: } & \text { (lançant un appel) } \\ \text { ni America kalu-o-e } & \text { mes hommes américains } \\ \text { gi wi o:ba-e } & \text { quels sont vos noms? } \\ \text { ni Australia gayo-o-e } & \text { mes femmes australiennes } \\ \text { gi wi o:ba-e } & \text { quels sont vos noms? } \\ \text { ni America kalu-o-e } & \text { mes hommes américains } \\ \text { wo: wo: } & \text { (appelant) } \\ \text { ni America kalu-o-e wo: wo: } & \text { mes hommes américains (appelant) } \\ \text { gi wi o:ba-e } & \text { quels sont vos noms? } \\ \text { ni Australia gayo-e } & \text { mes femmes australiennes } \\ \text { gi wi o:ba-e } & \text { quels sont vos noms? } \\ \text { ni America kalu-o-wo: } & \text { mes hommes américains } \\ \text { o wo:-wo: wo: } & \text { (appelant) } \\ \text { gi wi o:ba-e } & \text { quels sont vos noms? } \\ \text { ni Australia gayo-e } & \text { mes femmes australiennes } \\ \text { ni America kalu-o-e } & \text { mes hommes américains } \\ \text { a:-ye-wo: wo: } & \text { (appelant) }\end{array}$


Tandis que le magnétophone continuait d'enregistrer, Ulahi fit une pause, puis continua dans une voix chantée-parlée : « Mes hommes américains... wo: wo: ». Puis elle reprit une pleine voix parlée, continuant ainsi (traduction plutôt littérale) :

Eh bien, moi, j'y pense, j'en parle avec tristesse, je ne verrai pas ton lieu mais toi tu vois là où je vis, je ne connais pas tes noms, qui es-tu ? Je me demande, je pense comme ça, vous les gens qui vivez dans des pays lointains, qui m'écoutez, je n'ai pas entendu les noms de votre pays, alors qui êtes-vous ? C'est bien ce que je dis. Steve, toi qui es venu avant, tu peux dire, « mon nom est Steve, homme américain », mais tous les autres, quels sont vos noms ? «Beaucoup de gens entendront tes chants Bosavi », tu m'as dit ça comme ça avant, mais quand j'y pense, quand je chante seule je me demande, quels sont vos noms ? C'est à ça que je pensais. Je ne connais pas vraiment les noms des pays, seulement Amérique, Australie, alors je chante tristement comme ça pour qu'ils puissent l'entendre.

Ces remarques sont nées d'une conversation qu'Ulahi et moi avions eue en marchant ensemble de Bolekini vers l'endroit du ruisseau Wo:lu où elle chanta ses chants ce jour-là. Ulahi me demanda pourquoi je voulais enregistrer ses chants de nouveau. Je lui répondis que cette fois-là, de nombreuses nouvelles personnes entendraient l'enregistrement. Je ne pouvais pas vraiment expliquer dans un cadre kaluli en quoi Voices of the Rainforest marquait un grand changement par rapport aux albums académiques à diffusion limitée que j'avais publiés auparavant (Feld, 1981, 1985). Cela n'aurait rien signifié de dire à Ulahi que l'enregistrement serait publié par Rykodisc, une entreprise indépendante importante, ou que Mickey Hart, le percussionniste célèbre du Grateful Dead, était son producteur, ou qu'il aurait de la publicité, une distribution et des ventes dans le monde entier. Et il était impossible d'expliquer que cela allait être le premier disque compact, un objet qu'aucun Kaluli n'avait jamais vu, entièrement dédié à la musique de Papouasie-Nouvelle-Guinée, ou qu'il serait vendu dans des centaines de magasins et joué par des centaines de chaînes radio. Ces sujets, personnes, entreprises, et publications, aussi importants qu'ils puissent être pour des chercheurs ou le grand public, étaient entièrement étrangers au monde vécu quotidien kaluli. Aussi continuais-je à dire à Ulahi que beaucoup, beaucoup de gens en Amérique et en Australie, les deux endroits dont les Kaluli ont entendu parler à travers le contact avec des étrangers, entendraient sa voix et seraient très heureux de cela (à propos 
de l'esthétique de l'enregistrement, ainsi que des questions éthiques autour des droits d'auteur et de la politique de propriété, voir Feld, 1992).

Naturellement, le sentiment d'émerveillement et d'étrangeté suscités par cette conversation subsistait en Ulahi tandis qu'elle chantait son gisalo. Sa manière de poursuivre le dialogue émergea dans son bref chant improvisé ko:luba. En chantant dans un registre grave, fluide, interrogateur, semi-parlé, Ulahi créait le contraste entre le caractère explicite des noms de lieux et le mystère des noms de personnes, considérés comme un nœud identitaire essentiel. Mais sa voix, appelant depuis le bord du ruisseau Wo:lu des hommes américains et des femmes australiennes, pourrait résonner ailleurs comme un exemple bouleversant des ethnoscapes de la « schizophonia ${ }^{20} \gg$ (Schafer, 1977), un bruit local tout droit sorti du poste radiocassette mondial de la « différence bannie » (Attali, 1985). Bien que le chant donne satisfaction à un narcissisme postmoderne qui ne peut voir qu'un monde de réflexions fragmentaires issues des miroirs qu'il a lui-même brisés, il y a quelque chose de délicieusement local et profondément ancré ici. Les noms de lieux étrangers soulignent ce que la poétique kaluli souligne toujours, à savoir que les sentiers chantés relient des vies et des souvenirs proches et lointains, qu'ils reconnaissent les sentiments simultanés de familiarité et de rupture, qui sont au cœur de la confrontation émotionnelle avec la perte humaine.

L'usage de noms de lieux extérieurs n'est pas une pratique d'opposition ici, ni une tentative de subvertir ou de prendre le pouvoir sur d'autres qui dominent maintenant. Ce chant, à l'instar de tous les chants kaluli, initie une dialectique de l'emplacement et du déplacement et la résout dans une poétique du re-placement. Les Kaluli pratiquant cette esthétique du sentier chanté, les chanteurs dont les meilleurs chants font pleurer leurs auditeurs, sont terre-à-terre quand ils font appel à des noms de lieux lointains pour mettre en relief leurs propres pouvoirs d'évocation. Car au cœur de tout chant Kaluli, réside la création d'un espace-temps dialogique dans lequel imaginer les autres en chantant leurs terres permet d'inviter leur humanité et leur présence autant que leur altérité et leur absence. C'est la raison pour laquelle le chant d'Ulahi et son commentaire spontané expriment de manière si poignante cette interrogation, ce questionnement : quand on entend sa voix, pouvons-nous sentir à la fois son sentiment de reconnaissance et son sentiment de perte?

20. Schizophonia est un terme pour la première fois employé par R. M. Schafer pour désigner le décalage entre le son original et sa reproduction électro-acoustique. 


\section{Les lieux sont comme les membres d'une famille qui appellent}

Le troisième des chants de bord de ruisseau Wo:lu d'Ulahi est dans le genre heyalo, une forme structurée par l'alternance de mo:, le « tronc » ou refrain, et dun, des « branches », ou des couplets.
mo:
1
2
3
4

nimo sa:yalena: imolobo:

Wafulo: sa:iyalena: imolobo:

Weane Ho:iyo Wafulo:

nimo sa:yiyalena: imolobo:

$\begin{array}{lllll}\text { dun } & 1 & 2 & 3 & 4\end{array}$

kuguno: ge aowo: a:la:lubiyo:

Ho:iyo:wo: ge aowo: a:la:lubiyo:

Ho:bi Imano: Ho:bi

bolo: ge aowo: a:la:lubiyo:

a:ba:lo: bolo: bolo:

«tronc » 1

2

3

4

ma cigale d'eau a faim

au bord du Wafulo: la cigale est seule

Weane Ho:iyo:

ma cigale d'eau est abandonnée

«branches » 1

2

3

4

la vallée est comme ton frère qui appelle

A Ho:iyo: ton frère t'appelle

Ho:bi Imano: Ho:bi

près de l'arbre bol, ton frère qui appelle

a:ba:lo: bolo: bolo:

Le chant fait alterner quatre ensembles appariés de « troncs » et de «branches ». Chaque répétition $\mathrm{du}$ «tronc » change le nom de lieu d'une crête, répétant l'image initiale la dernière fois. Chaque répétition alternée des «branches » modifie le nom de lieu d'une montagne et d'un arbre qui s'y trouve, employant le même nom de lieu la deuxième et la quatrième fois et le même nom d'arbre la troisième et la cinquième fois. Remarquons l'absence de régularité dans la répétition des noms de lieux, de telle sorte que la répétition n'est pas trop prévisible.

Les lieux dans ce chant sont situés près de la rivière Dibano, loin au sud-est de la région où habitent les Kaluli. Wafulo:, Weane, Ho:iyo et Imane sont tous des hen dagon, des « crêtes de montagnes » au-dessus du fleuve Dibano, sur les terres 
du peuple Wo:siso:. Ho:bi est une montagne du côté du village de Walagu dans le pays Onabasulu, plus au nord. Les arbres mentionnés sont bol, un grand arbre fruitier à bois dur, et $a: b a: l$, un fin palmier ; les deux se tiennent toujours seuls. À ces lieux, le chant juxtapose des images visuelles et sonores de solitude, de faim, de sentiment de vide, et de liens familiaux. Ulahi dit que le pouvoir des « mots retournés » ou métaphoriques réside ici dans le fait que la faim, le sentiment de vide, est comme une voix qui vous appelle, ou comme une cigale bruyante près $\mathrm{du}$ courant. Comme le chuintement aigu de l'eau ou le gargouillis d'un estomac vide, c'est un son régulier, grinçant, glaçant qui ne veut pas s'en aller. Séjourner dans les vallées sous ces crêtes montagneuses est comme votre frère qui vous appelle. C'est parce que kugun, « la vallée forestière non déboisée », est comme un frère pour dagon, la «montagne ». Des lieux étroitement liés sont comme les membres d'une famille les uns pour les autres, de sorte que les reliefs qui en surplombent d'autres s'appellent l'un l'autre, désirant être reliés, comme des frères.

\section{«Comme une cascade dans ta tête »}

Pris comme un ensemble, les trois chants d'Ulahi dans le segment « Se détendre au ruisseau » illustrent les manières contrastées dont les sentiers des noms de lieux portent et expriment la biographie, les souvenirs, et les sentiments. Son chant gisalo parcourt des lieux relativement proches qui définissent en partie comment la vie d'une personne spécifique est liée à un sentiment d'inconfort dans son appartenance à la communauté et à ses marges. Comme le chant gisalo, le ko:luba d'Ulahi illustre le caractère central des noms de lieux dans la poesis de l'évocation et de la perte. Mais le chant va plus loin, en liant le pouvoir des noms de lieux au pouvoir des noms de personnes, pour créer un sentiment d'identité, proche ou loin, familier ou étranger. Et son chant heyalo chante un fragment d'images anciennes et lointaines, évoquant le souvenir d'une époque et d'un événement, les pensées d'autres personnes qui en furent émues, et la pensée selon laquelle en vivant loin, on se rappelle profondément des lieux en tant que liens de parenté ; les points sur le sentier sont comme des lieux familiers qui vous appellent en retour.

Un élément important a été jusqu'à présent mis de côté dans cette description des pouvoirs évocateurs de la dénomination des lieux et de la circulation de la mémoire des sentiers : la performance d'Ulahi elle-même. Pendant qu'elle chantait, Ulahi était assise sur la berge du $d u$ Wo:lu, un segment rocheux au milieu du ruisseau, avec son fils Wano, un de ses cochons, et moi. Mais elle ne chantait pas uniquement pour nous. Elle chantait avec le Wo:lu, elle chantait avec l'eau, elle chantait comme l'eau, elle chantait au sujet de l'eau. Il ne s'agissait pas d'un artifice pour l'enregistrement. Les hommes et les femmes kaluli composent 
souvent des chants près des ruisseaux et des cascades ; les femmes chantent souvent des chants dans des ruisseaux à proximité des sagoutiers ou autres cours d'eau.

Chanter comme l'eau est une idée qui réverbère à travers la langue et les pratiques d'expression Bosavi. Par exemple, le verbe pour « composer » en kaluli, sa-molab, implique d'entendre et de chanter à l'intérieur, « comme une cascade dans ta tête » ainsi que Jubi le dit un jour. Dans ce cas et dans d'autres, la puissance métaphorique de l'eau est indiquée par la polysémie de toute la terminologie liée à l'eau dans le champ sémantique du chant. On dit que composer un chant ressemble au flot d'une cascade dans un bassin d'eau. Quand les mots viennent à l'esprit et correspondent à la mélodie, c'est comme une cascade qui s'écoule dans un bassin, se retenant, restant, puis s'écoulant plus loin. Rappelons également que la partie centrale du chant gisalo, là où est établi son centre tonal, est appelée « chute d'eau ». C'est l'endroit où il résonne pour former un appel en $\ll \mathrm{o} »$ et une rime en $\ll \mathrm{e} »$ réverbérant en retour, et où il marque une pause avant d'atteindre ses « branches ». De même, le ou les deux derniers vers du gisalo sont le « bourdonnement de la cascade », là où le chant est emporté par de bourdonnants $\ll \mathrm{o} »$ et $\ll \mathrm{e} »$, de nouveau dans le centre tonal. Et en plus de ces manières dont les mélodies coulent comme de l'eau, le temps et la rythmicité du chant sont aussi des formes d'écoulement; la terminologie du rythme musical et du mètre est polysémique avec les qualités de battement, de jaillissement, et de mouvement de l'eau.

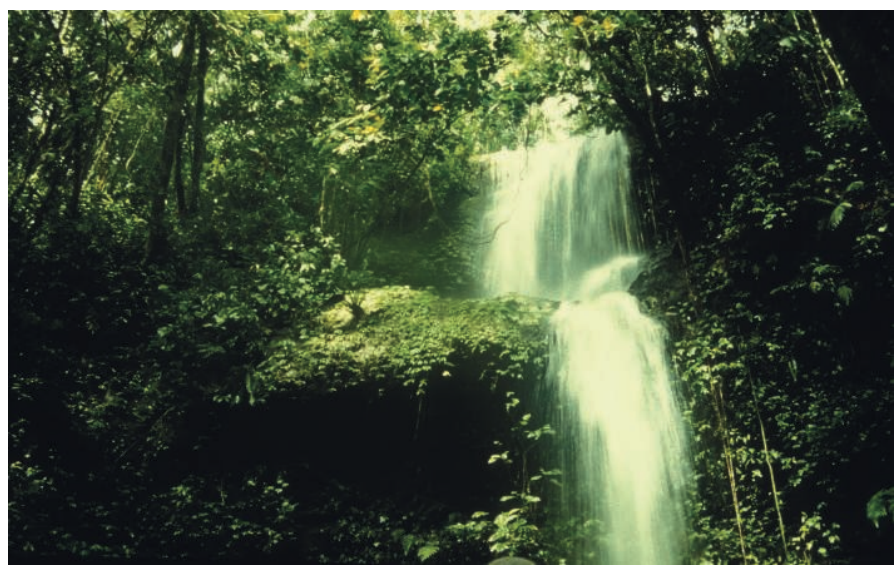

Figure 3

Le foo- jaillissant de la cascade So:lo: qui forme le sommet de la chanson gisalo d'Ulahi Photographie @ Steven Feld 
L'eau qui s'écoule à travers la terre, incorporée dans l'expérience vécue comme le flot cadencé de la voix à travers le corps qui résonne, irrigue l'imagination et la pratique du chant, depuis sa structure mélodique et rythmique jusqu'à ses qualités de timbre et de texture et son pouvoir évocateur à rester en tête. De la sorte, chanter avec l'eau dans un écoulement performatif, et chanter comme l'eau par la musicalité, sont en relation profonde avec le fait de chanter à propos de l'eau grâce à la localisation créée par le texte, de suivre des cours d'eau, d'évoquer leur présence sonique à travers des idéophones phonesthétiques. Un jour, Ulahi m'a dit que chacun de ses chants était comme un mogan sur un ruisseau. J'en comprends que, pour elle, chaque chant est un bassin, un tourbillon, quelque chose qui se rassemble et tourne en cercle sur place pour quelques instants, puis tourne et s'écoule vers l'aval pour se mêler et fusionner dans d'autres bassins. Les chants d'Ulahi, comme le ruisseau Wo:lu où elle les a chantés, ondoient et coulent à travers les communautés bosavi, se réverbérant à travers les vies kaluli et les nôtres en reliant des lieux et en suggérant que ces sentiers associent toujours des histoires ayant trait aux souvenirs et aux ressentis des gens.

\section{Lieu, expérience, expression}

«Si la perception mesure le pouvoir réflecteur du corps, l'affection en mesure le pouvoir absorbant »(Bergson, 1988, p. 56). Les lieux peuvent devenir présents à travers l'expérience de la sensation corporelle, mais c'est à travers l'expression qu'ils atteignent des dimensions émotionnelles et esthétiques accrues d'inspiration sensuelle. Le sens de l'emplacement kaluli implique la naturalisation sensorielle des manières dont les cours d'eau s'écoulent pour se mouvoir dans et à travers les terres, les manières dont la terre est toujours dans et autour de l'eau. Cette localisation est mise en œuvre par analogie corporelle : la voix se déplace dans et à travers le corps, mais la présence physique et émotionnelle du corps entier est toujours dans la voix. Reliant ensemble ce que Bergson appelle les pouvoirs 《réflecteur » et « absorbant » du corps, les Kaluli chantent au sujet des cours d'eau, chantent avec l'eau, imaginent que le chant est comme de l'eau qui s'écoule, semblable à la voix incorporée. Ici, la poétique du lieu se fond avec la volupté du lieu comme soundscape et avec la sensualité de la voix chantante.

Mais il y a plus encore : l'écoulement, le registre perpétuel d'images en résonance de l'eau, charrie et persiste dans la mémoire incorporée au-delà de l'immédiateté perceptive d'une expression vocale lors d'une performance. De sorte que, lorsqu'on se tient près d'un cours d'eau forestier puis qu'on continue de marcher dans la forêt, on entend la manière dont l'eau poursuit son cours, et 
on sait ainsi qu'elle peut s'écouler de nouveau dans une immédiateté perceptive aussi aisément qu'elle en est sortie. Cela reprend concrètement un thème d'Edward Casey dans son essai pour cet ouvrage ${ }^{21}:$ «Des corps qui perçoivent sont des corps qui savent, et, inséparable de ce qu'ils savent, est la culture telle qu'elle imprègne et donne forme à des lieux particuliers. C'est par les corps que les lieux deviennent des entités culturelles » (Casey, dans cet ouvrage ; voir aussi Casey, 1987, p. 146-180, 1993, p. 43-105). Cela revient à dire que les lieux font sens en grande partie grâce à la manière dont ils sont rendus sensuels et dont ils sont sensuellement exprimés par la voix. Les pratiques poétiques et performatives donnent une place centrale aux sens, en créant un sens local du lieu. C'est ainsi qu'une poétique découle d'une expérience quotidienne, et que l'imagination et les pratiques kaluli font de l'eau une acoustémologie de la résonance incorporée du lieu.

\section{Note de l'auteur}

Les mots kaluli sont orthographiés selon l'orthographe que l'on retrouve dans Feld, 1990, p. 17-19. Pour faciliter la lecture ici, o: signifie o phonétique ouvert, prononcé comme la voyelle dans bought en anglais, et a: équivaut au epsilon phonétique, prononcé comme la voyelle dans bet. D'autres voyelles sont orthographiées pour se rapprocher d'équivalents phonétiques anglais proches : i comme dans beet, u comme dans boot, e comme dans bait, o comme dans boat, a comme dans bob. Les consonnes, de même, sont orthographiées pour se rapprocher d'équivalents phonétiques anglais proches. La recherche sur laquelle est fondé cet essai fut entreprise en 1976-1977, 1982, 1984, 1990 et 1992, et approfondie en 1994-1995 dans les communautés kaluli de Sululeb et Bolekini au Bosavi, Papouasie-Nouvelle-Guinée. Je remercie tous mes hôtes, particulièrement Jubi, Kulu, Ayasilo, Ho:nowo:, Hobole, Hasele, Gigio, Ha:gulu, Deina, Seyaka et Ulahi pour leurs années d'amitié et leurs efforts pour rendre leur monde peuplé de lieux compréhensible pour moi. Je dois également beaucoup à Bambi B. Schieffelin et Edward L. Schieffelin pour des années de linguistique et d'ethnographie polyphoniques. Mon interprétation des sentiers de noms de lieux dans les trois chansons d'Ulahi provient en grande partie de discussions pendant et après les sessions d'écoute des cassettes et de transcription-traduction à Bolekini en août 1990 et en juillet et août 1992. À ces occasions, j'ai travaillé étroitement avec Ulahi et Ayasilo et j'ai également été aidé par Deina, Hobole et Hasele. Le soutien à la recherche a été généreusement pourvu par la Division des Études culturelles de l'Institut national de recherche de Papouasie-Nouvelle-Guinée, et aux États-Unis par la National Science Foundation, le National Endowment for the Arts, le National Endowment for the Humanities, la Wenner-Gren Foundation for Anthropological Research, Rykodisc, $360^{\circ}$ Productions, et la John D. and Catherine T. MacArthur Foundation. Pour leurs commentaires et leurs critiques sur cet essai, je remercie Keith Basso, Edward Casey, Alison Leitch, Bambi B. Schieffelin,

21. Senses of Place, l'ouvrage collectif dont est extrait le présent texte. 
Edward L. Schieffelin, Alan Rumsey, Lowell Lewis, Andrew Lattas, et Jadran Mimica. Pour leurs remarques additionnelles durant des cours à l'Université d'Oslo en mai 1993, j'ai de la reconnaissance pour Odd Are Berkaak, Vigdis Broch-Due, Arve Sørum, et Graham Townsley. Je remercie aussi les participants de Tuning of the World Conference on Acoustic Ecology à Banff, au Canada, en août 1993 pour avoir répondu avec une grande musicalité à une présentation sur l' « échomusécologie » et l'acoustémologie Bosavi.

\section{Bibliographie et discographie}

Abu-Lughod Lila \& Lutz Catherine, 1990, "Introduction: Emotion, Discourse, and the Politics of Everyday Life" in Language and the Politics of Emotion, vol. 1, Cambridge University Press, New York, p. 123.

Ackerman Diane, 1990, A Natural History of the Senses, Random House, New York, $331 \mathrm{p}$.

Attali Jacques, 1985 [1977], Noises: the Political Economy of Music, University of Minnesota Press, Minneapolis, 179 p.

Basso Ellen B., 1985, A Musical View of the Universe: Kalapalo Myth and Ritual Performances, University of Pennsylvania Press, Philadelphia, 356 p.

BAsso Keith, 1988, “'Speaking with names': Language and Landscape among the Western Apache” in Cultural Anthropology, vol. 3, n², pp. 99-130.

Bergson Henri, 1988 [1908], Matter and Memory, Zone Books, New York, $288 \mathrm{p}$.

Bourdieu Pierre, 1977 [1972], Outline of a Theory of Practice, Cambridge University Press, Cambridge, 248 p.

Buttimer Anne \& Seamon David (eds.), 1980, The Human Experience of Space and Place, St. Martins Press, New York, 204 p.

Carpenter Edmund, 1960, "Acoustic space" in Carpenter Edmund \& Mcluhan Marshall (eds.), Explorations in communication: An anthology, Beacon Press, Boston, pp. 65-70. 
Carpenter Edmund, 1971, "The Eskimo Artist" in Otten Charlotte (ed.), Anthropology and Art: Readings in CrossCultural Aesthetics, Natural History Press, New York, pp. 163-171.

Carpenter Edmund, 1973, Eskimo Realities, Holt, Rinehart and Winston, New York, $212 \mathrm{p}$.

Carpenter Edmund, 1980, "If Wittgenstein Had Been an Eskimo. Even for Profound Philosophers, Literacy Has its Limitations" in Natural History, vol. $89, \mathrm{n}^{\circ} 2$, pp. $72-77$.

Casey Edward S., 1987, Remembering: A Phenomenological Study, Indiana University Press, Bloomington, $362 \mathrm{p}$.

CASEY Edward S., 1993, Getting back into Place: Toward a Renewed Understanding of the Place-World, Indiana University Press, Bloomington, 403 p.

Cosgrove Denis E. (ed.), 1984, Social Formation and Symbolic Landscape, Croom Helm, London, 293 p.

Cyтowic Richard, 1989, Synesthesia: A Union of the Senses, SpringerVerlag, Berlin, $354 \mathrm{p}$.

Feld Steven, 1981, Music of the Kaluli, Institute of Papua New Guinea Studies, LP, Boroko.

Feld Steven, 1982, Sound and Sentiment: Birds, Weeping, Poetics, and Song in Kaluli Expression, University of Pennsylvania Press, Philadelphia, 264 p.

Feld Steven, 1985, Kaluli Weeping and Song, Barenreiter Musicaphon, LP, Kassel.

Feld Steven, 1986, "Orality and Consciousness" in Tokumaru Yoshihiko \& Yamaguchi Osamu (eds.), The Oral and the Literate in Music, Academia Music, Tokyo, pp. 18-27.

Feld Steven, 1988, "Aesthetics as Iconicity of Style, or 'Lift-up-over Sounding': Getting into the Kaluli Groove" in Yearbook for Traditional Music, vol. 20, pp. 74-113. 
Feld Steven, 1990, Sound and Sentiment: Birds, Weeping, Poetics, and Song in Kaluli Expression, $2^{\text {nd }}$ edition, University of Pennsylvania Press, Philadelphia, 297 p.

FELD Steven, 1991, Voices of the Rainforest, CD/cassette, Salem, Rykodisc, Massachusetts.

FELD Steven, 1992, "Voices of the Rainforest: 'Imperialist Nostalgia' and the Politics of Music" in Arena, n 99100, pp. 164-177.

Finnegan Ruth, 1988, Literacy and Orality, Basil Blackwell, Oxford, 256 p.

Gonzalez-Crussi Frank, 1989, The Five Senses, Vintage, New York, 162 p.

Heidegger Martin, 1971, Poetry, Language, Thought, Harper and Row, New York, 229 p.

Higgins Kathleen Marie, 1991, The Music of Our Lives, Temple University Press, Philadelphia, $258 \mathrm{p}$.

Howes David, 1991, The Varieties of Sensory Experience: A Sourcebook in the Anthropology of the Senses, University of Toronto Press, Toronto, $336 \mathrm{p}$.

IHDE Don, 1976, Listening and Voice: Phenomenologies of Sound, Ohio University Press, Athens, 188 p.

JaCKson Michael, 1989, Paths toward a Clearing. Radical Empiricism and Ethnographic Inquiry, Indiana University Press, Bloomington, 256 p.

Leder Drew, 1990, The Absent Body, University of Chicago Press, Chicago, $229 \mathrm{p}$.

Levin David Michael, 1985, The Body's Recollection of Being, Routledge \& Kegan Paul, London, 384 p.

MARKs Lawrence, 1978, The Unity of the Senses: Interrelations among the Modalities, Academic Press, New York, 302 p. 
Mauss Marcel, 1979 [1936], “Body Techniques” in Sociology and Psychology, Routledge and Kegan Paul, London.

Merleau-Ponty Maurice, 1962 [1945], Phenomenology of Perception, Humanities Press, New York, 466 p.

Merleau-Ponty Maurice, 1963 [1942], The Structure of Behavior, Beacon Press, Boston, $256 \mathrm{p}$.

Merleau-Ponty Maurice, 1964 [1947], The Primacy of Perception, Northwestern University Press, Evanston, Illinois, 228 p.

Ochs Elinor \& Schieffelin Bambi B., 1983, Acquiring Conversational Competence, Routledge, London, $224 \mathrm{p}$.

Ochs Elinor \& Schieffelin Bambi B., 1984, "Language acquisition and socialization: Three Developmental Stories and Their Implications" in Schweder Richard \& Levine Robert (eds.), Culture Theory: Essays on Mind, Self, and Emotion, Cambridge University Press, New York, pp. 276-320.

Ohnuki-Tierney Emiko, 1991, "Embedding and Transforming Polytrope: The Monkey as Self in Japanese Culture" in Fernandez James W. (ed.), Beyond Metaphor. The Theory of Tropes in Anthropology, Stanford University Press, Stanford, California, pp. 159-189.

Ong Walter J., 1982, Orality and Literacy, Methuen, New York, 201 p.

Sataloff Robert, 1992, "The Human Voice" in Scientific American, vol. 267, n 6 , pp. 108-115.

Schafer R. Murray, 1977, The Tuning of the World: Toward a Theory of Soundscape Design, Knopf, New York, 301 p.

Schafer R. Murray, 1985, "Acoustic space" in SeAmon David \& Mugerauer Robert (dir.), Dwelling, Place and Environment, Springer, Dordrecht, pp. 87-98.

SChafer R. Murray, 1993, Voices of Tyranny: Temples of Silence, Arcana Editions, Indian River, Ontario, 167 p. 
Schieffelin Bambi B., 1979, "Getting It Together: An Ethnographic Approach to the Study of the Development of Communicative Competence" in Ochs Elinor \& Schieffelin Bambi B. (dir.), Developmental Pragmatics, Academic Press, New York, pp. 73-108.

Schieffelin Bambi B., 1986, “The acquisition of Kaluli” in Slobin Dan (ed.), The CrossLinguistic Study of Language Acquisition, Erlbaum, Hillsdale, New Jersey, pp. 525-594.

Schieffelin Bambi B., 1990, The Give and Take of Everyday Life: Language Socialization of Kaluli Children, Cambrige University Press, New York, 288 p.

Schieffelin Edward L., 1976, The Sorrow of the Lonely and the Burning of the Dancers, St. Martins Press, New York, 223 p.

Schieffelin Edward L., 1977, "The Unseen Influence: Tranced Mediums as Historical Innovators" in Journal de la Société des Océanistes, vol. 33, n 56, pp. 169-178.

Schieffelin Edward L., 1982, “The bau a Ceremonial Hunting Lodge: An Alternative to Initiation” in HERDT Gilbert (dir.), Rituals of Manhood: Male Initiation in Papua New Guinea, University of California Press, Berkeley, pp. 155-200.

Schieffelin Edward L., 1984, "The Retaliation of the Animals: On the Cultural Construction of the Past in Papua New Guinea” in Bikmaus, vol. 5, $\mathrm{n}^{\circ} 4$, pp. 114.

SChieffelin Edward L., 1985, "Performance and the Cultural Construction of Reality” in American Ethnologist, vol. 12, n 4, pp. 707-724.

Schieffelin Edward L \& Crittenden Robert (dir.), 1991, Like People You See in a Dream: First Contact in Six Papuan Societies, Stanford University Press, Stanford, $328 \mathrm{p}$.

Schieffelin Edward L. \& Kurita Hiroyuki, 1988, “The Phantom Patrol: Reconciling Native Narratives and Colonial Documents in Reconstructing the History of Exploration in Papua New Guinea" in The Journal of Pacific History, vol. 23, $\mathrm{n}^{\circ}$ 1, pp. 52-69. 
Schilder Paul, 1950, The Image and Appearance of the Human Body, International Universities Press, New York, 353 p.

Seamon David, 1979, A Geography of the Lifeworld: Movement, Rest, and Encounter, St. Martins Press, New York, 227 p.

Seamon David \& Mugerauer Robert (eds.), 1985, Dwelling, Place and Environment: Towards a Phenomenology of Person and World, M. Nijhoff, Dordrecht, $310 \mathrm{p}$.

Sørum Arve, 1989, The Aesthetics of Spacetime: Temporality and Perception in New Guinea, article présenté lors de la rencontre annuelle de l'American Ethnological Society.

Stoller Paul, 1989, The Taste of Ethnographic Things: the Senses in Anthropology, University of Pennsylvania Press, Philadelphia, 200 p.

Straus Erwin, 1963, The Primary World of Senses: A Vindication of Sensory Experience, Free Press, Glencoe, Illinois, 428 p.

TAussig Michael, 1993, Mimesis and Alterity: A Particular History of the Senses, Routledge, New York, 320 p.

Truax Barry, 1984, Acoustic Communication, Ablex, Norwood, New Jersey, $244 \mathrm{p}$.

Tuan Yi-Fu, 1977, Space and Place: The Perspective of Experience, University of Minnesota Press, Minneapolis, $248 \mathrm{p}$.

Urban Greg, 1991, A Discourse-Centered Approach to Culture, University of Texas Press, Austin, $215 \mathrm{p}$.

Varela Francisco, Thompson Evan \& Rosch Eleanor, 1991, The Embodied Mind: Cognitive Science and Human Experience, MIT Press, Cambridge, Massachussetts, $328 \mathrm{p}$.

Wagner Roy, 1986, Symbols that Stand for Themselves, University of Chicago Press, Chicago, 157 p. 
Weiner James, 1991, The Empty Place: Poetry, Space, and Being Among the Foi of Papua New Guinea, University of Indiana Press, Bloomington, 218 p.

WERner Hans Ulrich, 1992, Soundscapesakustische Landschaften: eine klangökologische Spurensuche, Soundscape Newsletter (Europe), Bâle, 282 p.

Zuckerkandl Victor, 1956, Sound and Symbol: Music and the External World, Bollingen Series, n 44, Princeton University Press, New Jersey, 407 p.

Résumé : Dans ce célèbre article initialement paru en 1996 et présenté ici en version française, Steven Feld introduit le concept d' « acoustémologie », ou connaissance par le son. Après avoir montré en quoi la recherche sur l'expérience acoustique et l'expression du lieu est demeurée relativement peu développée, l'auteur présente le monde sonore kaluli. Il propose ensuite deux parties ethnographiques portant sur l'acoustémologie de l'écoulement. La première traite des pratiques de dénomination kaluli, afin de montrer comment le caractère inséparable des eaux et des terres de la forêt tropicale est expérimenté et imaginé comme le flot de la voix à travers les contours du corps. Ce trope de l'écoulement est alors étudié tel qu'il apparaît dans des textes poétiques de chants, dans lesquels le fait de chanter une séquence de noms de lieux emmène les auditeurs dans un voyage qui s'écoule le long des cours d'eau et à travers les terres locales. L'écoulement de ces sentiers poétiques chantés est lié émotionnellement et physiquement au flot sensuel de la voix chantante. La mise en lien de ces sentiers qui s'écoulent révèle une acoustémologie kaluli des relations aux lieux, une fusion de l'espace et du temps qui assemble les vies et les événements en tant que souvenirs incorporés. Les pouvoirs évocateurs de cette acoustémologie atteignent un sommet esthétique dans la performance poétique, là où le flot expressif de la voix fusionne avec l'écoulement vécu des noms de lieux chantés, afin de créer des chants en cascade, un sens de la résonance du lieu.

Mots-clefs : acoustémologie, ethnomusicologie, anthropologie, sens, chant, lieu, son, écoulement, flot, sentier, genre, poésie, performance, Bosavi, Kaluli, Papouasie-Nouvelle-Guinée.

\section{Waterfalls of Song. An Acoustemology of Place Resounding in Bosavi, Papua New Guinea}

Abstract: In this study, Steven Feld investigates the sense of place of Kaluli people in Bosavi, Papua New Guinea. Because sound and an ear and voice centered sensorium are central to Kaluli experience and expression in the tropical rainforest, 
the goal of this exploration is to interpret what Feld calls an acoustemology, by which he means local conditions of acoustic sensation, knowledge, and imagination embodied in the culturally particular sense of place resounding in Bosavi. The chapter opens with brief notes on sensation, sound, synesthesia, and soundscapes that provide context for the general framework of the inquiry. He outlines ways in which research on acoustic experience and expression of place has remained relatively underdeveloped and then introduces the sound world of the Kaluli. Next, he offers two ethnographic sections on the acoustemology of flow. The first treats Kaluli naming practices to show how the inseparability of rainforest waters and lands is encountered and imagined to be like the flow of voice through the body's contours. This trope of flow is then examined as it appears in poetic song texts, where singing a sequence of named places takes listeners on a journey that flows along local waterways and through local lands. The flow of these poetic song paths is emotionally and physically linked to the sensual flow of the singing voice. Connecting these flowing paths reveals a Kaluli acoustemology of place relations, a fusion of space and time that joins lives and events as embodied memories. The evocative powers of this acoustemology reach an aesthetic apex in poetic performance, where the expressive flow of the voice merges with the experiential flow of sung placenames to create waterfalls of song, a sense of place resounding.

Keywords: acoustemology, ethnomusicology, anthropology, sense, song, place, sound, flow, path, gender, poetry, performance, Bosavi, Kaluli, Papua New Guinea

\section{Notes sur l'auteur}

Les travaux académiques de Steven Feld portent essentiellement sur l'anthropologie du son, une expression qu'il a créée en 1972 afin d'ouvrir l'anthropologie de la musique et du langage à une dimension plus sensorielle et esthétique, incluant la voix et la poétique, les relations au son de toutes les espèces, les médias et les technologies et l'environnement acoustique et écologique. 